Cite this: Phys. Chem. Chem. Phys., 2014, 16, 4917

Received 6th November 2013, Accepted 14th January 2014

DOI: $10.1039 / \mathrm{c} 3 \mathrm{cp} 54707 \mathrm{~h}$

www.rsc.org/pccp

\section{Effects of architecture on the stability of thermosensitive unimolecular micelles $\dagger$}

\author{
Alexander A. Steinschulte, ${ }^{a}$ Bjoern Schulte, ${ }^{b}$ Stephan Rütten, ${ }^{b}$ Thomas Eckert, ${ }^{a}$ \\ Jun Okuda, ${ }^{c}$ Martin Möller, ${ }^{b}$ Stefanie Schneider, ${ }^{a}$ Oleg V. Borisov ${ }^{\text {def }}$ and \\ Felix A. Plamper ${ }^{\star a}$
}

The influence of architecture on polymer interactions is investigated and differences between branched and linear copolymers are found. A comprehensive picture is drawn with the help of a fluorescence approach (using pyrene and 4HP as probe molecules) together with IR or NMR spectroscopy and X-ray/ light scattering measurements. Five key aspects are addressed: (1) synergistic intramolecular complexation within miktoarm stars. The proximity of thermoresponsive poly(propylene oxide) (PPO) and poly(dimethylaminoethyl methacrylate) (PDMAEMA) within a miktoarm star leads to complexation between these weakly interacting partners. Consequently, the original properties of the constituents are lost, showing hydrophobic domains even at low temperatures, at which all homopolymers are water soluble. (2) Unimolecular micelles for miktoarm stars. The star does not exhibit intermolecular selfassembly in a large temperature range, showing unimers up to $55^{\circ} \mathrm{C}$. This behavior was traced back to a reduced interfacial tension between the PPO-PDMAEMA complex and water (PDMAEMA acts as a "microsurfactant"). (3) Unimolecular to multimolecular micelle transition for stars. The otherwise stable unimolecular micelles self-assemble above $55{ }^{\circ} \mathrm{C}$. This aggregation is not driven by PPO segregation, but by collapse of residual PDMAEMA. This leads to micrometer-sized multilamellar vesicles stabilized by poly(ethylene oxide) (PEO). (4) Prevention of pronounced complexation within diblock copolymers. In contrast to the star copolymers, PPO and PDMAEMA adapt rather their homopolymer behavior within the diblock copolymers. Then they show their immanent LCST properties, as PDMAEMA turns insoluble at elevated temperatures, whereas PPO becomes hydrophobic below room temperature. (5) Two-step micellization for diblock copolymers. Upon heating of linear copolymers, the dehydration of PPO is followed by self-assembly into spherical micelles. An intermediate prevalence of unimolecular micelles is revealed in a small temperature window between PPO collapse and self-assembly of PEO- $b$-PPO. Also for PPO- $b$-PDMAEMA, PPO segregation prevails after initial weak complexation, leading to micelles with a PPO core. Considerable amounts of water are entrapped within the collapsed PDMAEMA domains above $55{ }^{\circ} \mathrm{C}$ (skin effect), preventing PPO-PDMAEMA complexation within precipitating PPO-bPDMAEMA. Further, collapsed PDMAEMA is rather polar as sensed by pyrene and 4HP. In summary, advanced macromolecular architectures can lead to an unprecedented intramolecular self-assembly behavior, where internal complexation prevents intermolecular aggregation.

\footnotetext{
${ }^{a}$ Institute of Physical Chemistry, RWTH Aachen University, Landoltweg 2, 52056 Aachen, Germany. E-mail: plamper@pc.rwth-aachen.de; Fax: +49 2418092327; Tel: +492418094761

${ }^{b}$ Interactive Materials Research - DWI an der RWTH Aachen e.V, Forckenbeckstraße 50, 52056 Aachen, Germany

${ }^{c}$ Institute of Inorganic Chemistry, RWTH Aachen University, Landoltweg 1, 52056 Aachen, Germany

${ }^{d}$ Institut Pluridisciplinaire de Recherche sur, l'Environnement et les Matériaux, UMR 5254 CNRS/UPPA, Pau, France

${ }^{e}$ Institute of Macromolecular Compounds of the Russian Academy of Sciences, 199004 St. Petersburg, Russia

${ }^{f}$ St. Petersburg National Research University of Information Technologies, Mechanics and Optics, Kronverkskiy pr., 49, 197101 St. Petersburg, Russia

$\dagger$ Electronic supplementary information (ESI) available: Experimental details of the preparation and characterization of the diblock copolymers, fluorescence spectroscopy, IR and NMR spectroscopy, scattering experiments, titration behavior and microscopy. Furthermore, discussion on the $c m c$ determination, the solubility of PPO, the general fluorescence behavior of the dyes, NOESY NMR, hysteresis, SAXS, cryo-SEM images of star aggregates and cryo-STEM images of the loose aggregates. See DOI: $10.1039 / \mathrm{c} 3 \mathrm{cp} 54707 \mathrm{~h}$
} 


\section{Introduction}

The formation of unimolecular micelles has been found in many polymeric systems. In most cases, a solvophobic interior of a centro-symmetric molecule is protected from the solvent by solvophilic moieties. Thus, star-shaped polymers with block copolymer arms and/or hydrophobic cores can lead to spherical unimolecular micelles in the case of a sufficiently high arm number. $^{1-10}$ Generally, other (e.g. worm-like) morphologies of unimolecular micelles could also be found, when using block copolymer side chains for molecular brushes. ${ }^{11}$ Block copolymer side chains are not necessarily a prerequisite for the formation of unimolecular micelles, since miktoarm stars (also called heteroarm stars) or even graft copolymers and multiblock copolymers can also suppress intermolecular aggregation. ${ }^{12-16}$ But in all cases, the number of solvophilic moieties compared to the area of the solvophobic/solvophilic interface needs to be sufficiently high, as seen for a decrease in the aggregation number with increasing arm number. ${ }^{17,18}$ In addition, only centro-symmetric molecules are reported to form unimolecular micelles with very few exceptions. ${ }^{19,20}$ Unimolecular micelles have been found in solutions of e.g. polyferrocenylsilane-based miktoarm stars in organic solvent. Here, the solvophilic arms were longer than the insoluble part in order to protect the segregated polymer chain from further aggregation. This architecture then leads directly to a Janustype of morphology, ${ }^{21}$ which has also been predicted by simulations of centro-symmetric systems. ${ }^{22,23}$ Very recently, we have also reported on non-centrosymmetric unimolecular micelles, which were formed upon stabilization against intermolecular aggregation by intramolecular complexation of different arms of the star-shaped molecules. ${ }^{20}$ We strengthened our findings by use of a scaling approach, showing that the reduction of interfacial tension upon complexation is one key feature for the generation of unimolecular micelles. ${ }^{20}$

Now within this article, the validity of our recent findings is corroborated by a broadened fluorescence spectroscopical approach together with further IR, NMR, X-ray and light scattering measurements to extract additional features of the self-assembly. Again, we choose to use poly(propylene oxide) (PPO; also called PPG: poly(propylene glycol)) as a hydrophobic arm, which is attached to the center of the star. Atactic linear PPO shows miscibility with water at low temperatures. Upon heating, it separates into a PPO-rich and a PPO-poor phase. ${ }^{24}$ This behavior can be termed an LCST-behavior (for Lower Critical Solution Temperature). The cloud points of PPO are dependent on the molecular weight and concentration. ${ }^{24}$ Oligomeric PPO is highly water-soluble (e.g. $\mathrm{PPO}_{7}$ has its LCST at approximately $55{ }^{\circ} \mathrm{C}$; the index assigns the number average degree of polymerization), whereas the LCST of $\mathrm{PPO}_{18}$ is already at $1{ }^{\circ} \mathrm{C}$. Therefore, medium-sized PPO is only miscible with water at low (and high) PPO content (e.g. $0.05 \mathrm{wt} \% \mathrm{PPO}_{52}$ yields a cloud point close to $15{ }^{\circ} \mathrm{C}$ ) and an LCST close to $-53{ }^{\circ} \mathrm{C}$ was estimated for high molecular weight PPO. ${ }^{24}$ Atactic PPO is otherwise a remarkable polymer, since it is highly soluble in most of the common organic solvents (ranging from very unpolar alkanes to the very polar cold water). PPO is best known for its amphiphilic, temperature-sensitive triblock copolymers (poloxamers) with ethylene oxide (branded e.g. as Pluronic $\left.^{\mathrm{TM}}\right) .{ }^{25}$ These triblock copolymers belong to the bestinvestigated polymer classes with numerous applications in cosmetics, drug delivery and so on. As an example, the thermoresponsive PPO-based micellization was followed by fluorescence studies, indicating a partial dehydration of PPO even below the critical micellization temperature $(\mathrm{cmt}) \cdot{ }^{26}$ Also NMR showed a cooperative preassociation and water-loss of PPO at temperatures lower than cmt, which will be shown by fluorescence spectroscopy in this work for related polymers. ${ }^{27}$ Different block copolymers were prepared with multiple thermosensitivity or multiple solvophilicity. ${ }^{28,29}$

Poly(dimethylaminoethyl methacrylate) (PDMAEMA) is the second polymer, which we incorporated into the miktoarm stars. Like PPO, PDMAEMA shows an LCST-behavior, which in contrast to PPO is very much pH-dependent (under the conditions used, PDMAEMA turns water insoluble at $\left.\sim 55{ }^{\circ} \mathrm{C}\right) .{ }^{30}$ Our group has prepared PDMAEMA-based copolymers with advanced architectures before. ${ }^{31-33}$ Furthermore, a number of amphiphilic PDMAEMA stars can be found in the literature. ${ }^{34,35}$ Recently, one of the first temperature-dependent fluorescence studies on mere PDMAEMA has been published. ${ }^{36}$ Interestingly, the fluorescence dye could be attached only to the polymer by ionic interactions and any dye loading by hydrophobic interactions is hardly detectable, since the dye is expelled from the polymer upon phase separation. A similar dye showed a combined electrostatically and hydrophobically induced binding, but temperature dependent data are missing for that particular dye. ${ }^{37}$ A similar picture was drawn for other PDMAEMA structures, where the polarity does not change upon phase separation (despite changes in microviscosity around the probe as seen from temperature-dependent fluorescence intensity). ${ }^{31,38}$ This unexpected behavior was also seen in this work and more work needs to be done in order to investigate the peculiar LCST-type phase-separation of PDMAEMA.

To prevent precipitation of the aggregates upon PDMAEMA collapse, we introduced a third polymeric component into our stars. One poly(ethylene oxide) arm (PEO; also called PEG: poly(ethylene glycol)) was attached onto the core of the star to assure colloidal stability by steric repulsion of the self-assembled aggregates at high temperatures. Furthermore, it can act as a reference for NMR, since PEO is highly water-soluble in the accessible temperature region. ${ }^{20,24}$ Nevertheless, we mainly concentrate our research below the phase-transition of PDMAEMA.

In literature, PPO- and PDMAEMA-based copolymers are known. ${ }^{39,40}$ But only recently, special interaction of PPO and PDMAEMA was reported, leading to miscibility in bulk. ${ }^{41}$ Weak attractive forces between both constituents seem to be responsible for this behavior, since the complexation of PPO with PDMAEMA in aqueous solution was so far unknown. ${ }^{20}$ As already illustrated in a former communication, ${ }^{20}$ higher segment densities of PDMAEMA around PPO are required in order to observe complex formation in the dilute regime. In bulk, the close vicinity between PDMAEMA and PPO units is ensured, but in solution special measures need to be taken in order to 
observe the interplay between both polymers. As an example, miktoarm star polymers are possible candidates in order to increase the segment density of one polymer constituent around the partner. The segment density within the star is higher than in linear copolymers, especially close to the core of the star. This can render the properties of one polymer block quite different.

In analogy to a linear pendant ${ }^{29}$ we combine PPO, PDMAEMA and PEO into one polymer star, the synthesis of which was described in detail in a recent paper. ${ }^{42}$ Further, the behavior in solution, which was partly highlighted in a former publication, ${ }^{20}$ will be corroborated by further spectroscopic means and scattering experiments. For further clarification, we again compare the results to binary polymer fragments, which were "cut out" from the miktoarm star. Thus, diblock copolymers (like PPO- $b$-PEO and PPO- $b$-PDMAEMA) were prepared and analyzed together with a PDMAEMA-based miktoarm star without PPO.

\section{Experimental section}

Details of the samples and the experimental details can be found in the ESI. $\dagger$ The characteristics of the samples are summarized in Table 1.

\section{Results}

The investigation of the thermoresponsive properties of the miktoarm stars and their related diblocks was performed in aqueous, buffered solution. See Table 1 for the composition of the investigated samples. In all cases, our emphasis was laid on the temperature-dependent fluorescence spectroscopy of dye molecules as sensors for the presence of hydrophobic domains in the system. The evolution of size with temperature was traced using a combined DLS (dynamic light scattering) and SLS (static light scattering) approach. For a better understanding, we compared the results of the miktoarm stars with the results of the respective block copolymers (see Table 1 and ESI $\dagger$ for characterization and synthesis). The PPO concentration was kept

Table 1 Molecular characterization of the polymers used (miktoarm stars and diblock copolymers; general assignment: $A_{n}-\left(B_{o}\right)_{k}-C_{m}$ with $k B$ arms; $n, o$ and $m$ assign the degree of polymerization of each block)

\begin{tabular}{|c|c|c|c|}
\hline & $\begin{array}{l}M_{\mathrm{n}}(\text { theo }) \\
{\left[\mathrm{g} \mathrm{mol}^{-1}\right]}\end{array}$ & $\begin{array}{l}M_{n}(\mathrm{NMR}) \\
{\left[\mathrm{g} \mathrm{mol}^{-1}\right]}\end{array}$ & $\begin{array}{l}M_{n}(\mathrm{SEC}) \\
{\left[\mathrm{g} \mathrm{mol}^{-1}\right]\left(\bigoplus_{\mathrm{M}, \mathrm{SEC}}\right)}\end{array}$ \\
\hline $\begin{array}{l}\mathrm{PEO}_{114}-\left(\mathrm{PDMAEMA}_{90}\right)_{3.1}-\mathrm{PPO}_{69} \\
\mathrm{PEO}_{114}-\left(\mathrm{PDMAEMA}_{110}\right)_{2.5} \\
\mathrm{PPO}_{69}-b-\mathrm{PDMAEMA}_{100} \\
\mathrm{PEO}_{114}-b-\mathrm{PPO}_{69}\end{array}$ & $\begin{array}{l}55000^{a, d} \\
48000^{e} \\
20000^{a} \\
9000^{f}\end{array}$ & $\begin{array}{l}61000^{b, d} \\
- \\
20000^{b} \\
9000^{b}\end{array}$ & $\begin{array}{l}58900(1.39)^{c, d} \\
54000(1.18)^{e} \\
21900(1.10)^{c} \\
16900(1.10)^{c}\end{array}$ \\
\hline \multicolumn{4}{|c|}{$\begin{array}{l}{ }^{a} \text { Calculated from the molar ratio of monomer/initiation site times the } \\
\text { conversion (as seen by }{ }^{1} \mathrm{H}-\mathrm{NMR} \text { ); molar mass of the macroinitiator has } \\
\text { been added. }{ }^{b} \text { Calculated by }{ }^{1} \mathrm{H} \text { NMR assuming a molar mass of the } \\
\text { PEO moiety of } 5000 \mathrm{~g} \mathrm{~mol}^{-1} \text { (or } 4000 \mathrm{~g} \mathrm{~mol}{ }^{-1} \text { for PPO block for } \\
\text { PDMAEMA-based linear polymers). }{ }^{c} \text { Apparent molecular weight and } \\
\text { dispersity }\left(D_{\mathrm{M}}\right) \text { as determined by SEC in DMF and using PMMA } \\
\text { standards. }{ }^{d} \text { Taken from ref. } 42 .{ }^{e} \text { Taken from ref. } 32 .{ }^{f} \text { Sum of mole- } \\
\text { cular weight of the precursors. }\end{array}$} \\
\hline
\end{tabular}

$M_{\mathrm{n}}$ (theo) $M_{n}(\mathrm{NMR}) M_{n}(\mathrm{SEC})$

$\mathrm{PEO}_{114}\left(\mathrm{PDMAEMA}_{110}\right)_{2.5} \quad 48000^{e} \quad-\quad 54000(1.18)^{e}$ $\mathrm{PPO}_{69}-b$-PDMAEMA $100 \quad 20000^{a} \quad 20000^{b} \quad 21900(1.10)^{c}$ $\mathrm{PEO}_{114}-b-\mathrm{PPO}_{69}$ cular weight of the precursors. constant in most cases in order to facilitate comparison ( $\sim 1.2 \mathrm{mmol} \mathrm{L}{ }^{-1}$ for monomeric units; polymer concentration $\left.\sim 2 \times 10^{-5} \mathrm{~mol} \mathrm{~L}^{-1}\right)$.

Fluorescence spectroscopy is a powerful method to learn about intermolecular aggregation and micellization. ${ }^{43}$ We used two different dyes, whose emission spectra are sensitive to the polarity of the surrounding medium, in order to detect the presence of hydrophobic domains: pyrene and 4-(dicyanomethylene)2-methyl-6-( $p$-dimethylaminostyryl)- $4 H$-pyran (abbreviated here as $4 \mathrm{HP}$; another abbreviation is DCM). ${ }^{20}$ Pyrene is a prominent standard probe by taking the intensity ratio $I_{1} / I_{3}$ of the first and third vibronic emission bands. ${ }^{44}$ Large ratios $\left(I_{1} / I_{3}\right.$ close to 2$)$ reflect a hydrophilic and polar environment, whereas small values $\left(I_{1} / I_{3}\right.$ around 1$)$ indicate a non-polar, hydrophobic surrounding of the pyrene molecules. Therefore, pyrene is prominent for the determination of the critical micellization concentration (cmc). It is especially useful for the cmcdetermination of low molecular weight surfactants, but this method was also adapted for polymeric amphiphiles (see also discussion in the ESI $\dagger$ ). ${ }^{26}$ In continuation, we were more interested in the investigation of the critical micellization temperature $(\mathrm{cmt})$ than in the cmc. In most of our systems, a temperature-dependent appearance of hydrophobic domains is detected, which is not necessarily connected to an intermolecular micellization process. This will be discussed in detail further below.

It is known that pyrene is sensitive toward quenching, which can be induced by tertiary amines. ${ }^{45}$ Therefore, there is a demand for a fluorescence probe with less sensitivity towards tertiary amines in order to compare the results, which were obtained in the presence of polyamines. Therefore we selected $4 \mathrm{HP}$ as well. While having discussed the polarity sensitivity of $4 \mathrm{HP}$ before, ${ }^{20} 4 \mathrm{HP}$ is supposed to be also sensitive to microviscosity. ${ }^{46,47}$ The wavelength at the maximum of the emission spectrum $\lambda_{\max }$ correlates with the polarity (polar environment correlates with large $\lambda_{\max }$ ). The overall intensity of the emission band is said to be influenced by the microviscosity. In order to check the feasibility of $4 \mathrm{HP}$ and pyrene for our system, we have conducted a number of test experiments, in order to extract the general features of the dyes (ESI $\dagger$ ). It turned out that pyrene is still suitable for our experiments, though the fluorescence intensity decreased in the presence of amine-containing polymers. This partly explains quantitative differences between both dyes. Qualitatively, both dyes show a similar behavior as seen in the following: we compared the behavior of both dyes, in order to draw conclusions on the special interaction between PPO and PDMAEMA in the case of a miktoarm star.

Fig. 1 shows the response of pyrene and 4HP probes, which are solubilized in a $\mathrm{PEO}_{114}-b-\mathrm{PPO}_{69}$ solution. As expected, the fluorescent probes sense a hydrophilic environment at low temperatures, since PPO is water-soluble under these conditions. From $5{ }^{\circ} \mathrm{C}$ onwards, the PPO of the diblock turns hydrophobic. This effect is best seen for the 4HP probe, which has a sharp drop in the $\lambda_{\max }$-value around $5{ }^{\circ} \mathrm{C}$. At higher temperatures, the indicator of polarity moves again towards the 

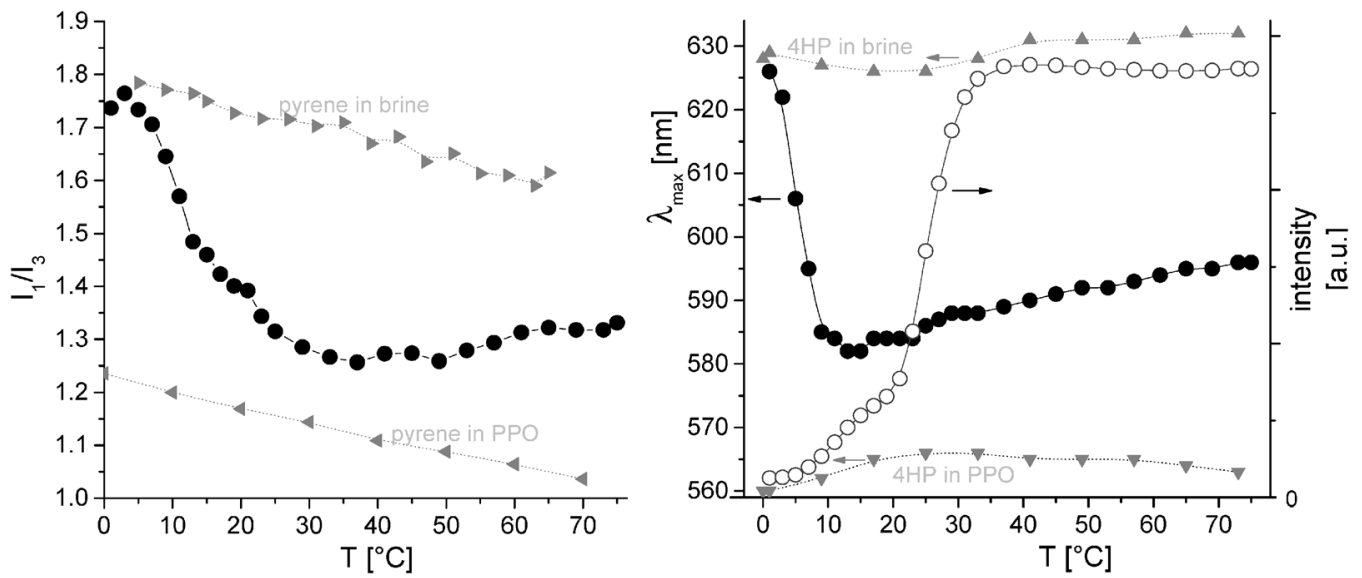

Fig. 1 Fluorescence spectroscopy of different probes in $\mathrm{pH} 8$ buffer ( $+0.1 \mathrm{M} \mathrm{NaCl}$ ) containing $0.16 \mathrm{~g} \mathrm{~L}^{-1} \mathrm{PEO}_{114}-b$-PPO 69 (except for the gray triangles); left hand side: intensity ratio of the first $\left(\lambda_{\mathrm{em}}=373 \mathrm{~nm}\right)$ and third vibronic fluorescence bands $\left(\lambda_{\mathrm{em}}=383 \mathrm{~nm}\right)$ of pyrene $\left(5 \times 10^{-7} \mathrm{M}\right.$ pyrene; $\lambda_{\text {exit }}=333 \mathrm{~nm}$, $\lambda_{\mathrm{em}}=340-500 \mathrm{~nm}$ ) in the presence of $\mathrm{PEO}_{114}-b-\mathrm{PPO}_{69}$ (black full circles) and in the absence of the diblock copolymer (gray triangles; directed toward the right: pyrene in pure aqueous solvent; directed toward the left: pyrene in pure PPO 4000 without water); right hand side: emission maximum $\lambda_{\text {max }}$ (black full circles) and intensity of the fluorescence peak (open circles) of $4 \mathrm{HP}$ as a probe $\left(10^{-6} \mathrm{M} ; \lambda_{\text {exit }}=470 \mathrm{~nm}, \lambda_{\mathrm{em}}=480-750 \mathrm{~nm}\right.$; heating $20 \mathrm{~K} \mathrm{~h} \mathrm{~h}^{-1}$; lines are guide to the eye); again, the $\lambda_{\max }$ of $4 \mathrm{HP}\left(10^{-6} \mathrm{M}\right)$ in pure solvent (upward directed gray triangles) and pure PPO 4000 in the absence of water (downward directed gray triangles) are indicated; part of data is taken from ref. 20 (reprinted with permission from ACS Macro Lett., $2012,1,504$. Copyright 2012 American Chemical Society).

hydrophilic side, which can be regarded as a consequence of a changing equilibrium distribution of the dye (Nernst partition law for dye solubilization either in the hydrophobic environment or in hydrophilic bulk solution). A more detailed thermodynamic analysis was given in our recent communication. ${ }^{20}$ Additionally, the temperature-dependent fluorescence intensity of $4 \mathrm{HP}$ shows a stepwise behavior, which can be hardly seen by the polarity-indicators $\left(I_{1} / I_{3}\right.$ and $\lambda_{\max }$, respectively). It can be interpreted in terms of an initial dehydration of PPO (probably forming "unimolecular micelles" with a blob of a partially collapsed PPO attached to a solvating PEO-block). The second step includes further aggregation, forming then spherical, starlike micelles and concomitantly changing the microviscosity within the PPO aggregate. The second step is close to the rapid increase in the number of micelles, which will be seen in a corroborating, detailed light scattering analysis as well (Fig. 5).

For comparison, we switched to the $\mathrm{PPO}_{69}-b$-PDMAEMA 100 diblock copolymer. Fluorescence spectroscopy detects again a change of solubilization of probe dyes, similarly to the one observed in Fig. 1. Despite a rather similar onset of hydrophobization, $\mathrm{PPO}_{69}-b$-PDMAEMA ${ }_{100}$ seems to be slightly more hydrophobic than $\mathrm{PEO}_{114}-b-\mathrm{PPO}_{69}$ (curves of $\mathrm{PPO}_{69}-b$-PDMAEMA 100 are below the curves of $\left.\mathrm{PEO}_{114}-b-\mathrm{PPO}_{69}\right)$. The dispersed, butylterminated PPO precursor (for the $\mathrm{PPO}_{69}-b$-PDMAEMA ${ }_{100}$ ) might play an additional role in this behavior. Again, $4 \mathrm{HP}$ is the first choice for probing hydrophobic environments (see Fig. 2; for measurements using pyrene, see the figure in the ESI $\dagger$ ), since here a two-step solubilization or micellization process is also resolved (first step at $5{ }^{\circ} \mathrm{C}$ and second one at $20^{\circ} \mathrm{C}$ ). Again, it can be interpreted in terms of an initial dehydration (still unimolecularly dispersed polymer), which is followed by further aggregation at higher temperatures. The main difference compared to $\mathrm{PEO}_{114}-b-\mathrm{PPO}_{69}$ is the minimum in $\lambda_{\max }$ between 6 and $16{ }^{\circ} \mathrm{C}$.

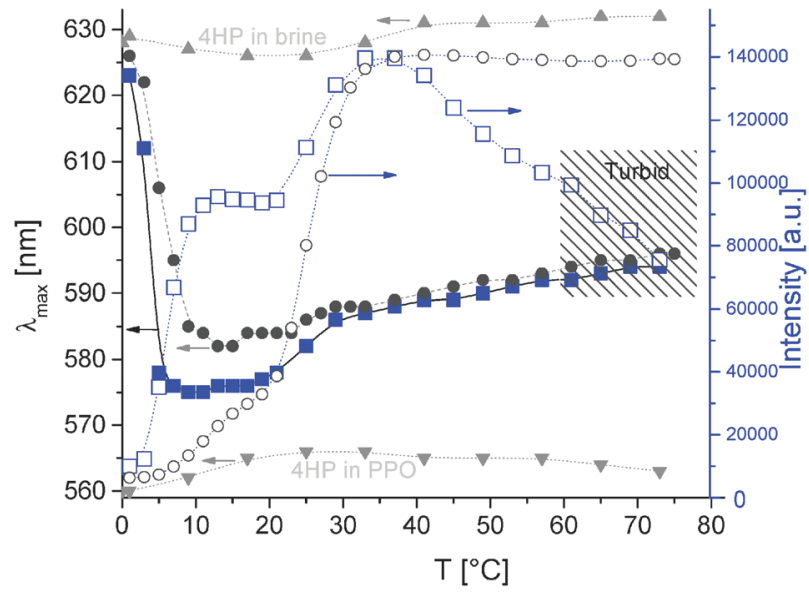

Fig. 2 Fluorescence spectroscopy of $4 \mathrm{HP}$ in $\mathrm{pH} 8$ buffer (+0.1 $\mathrm{M} \mathrm{NaCl}$ ) containing $0.4 \mathrm{~g} \mathrm{~L}^{-1} \mathrm{PPO}_{69}-b-\mathrm{PDMAEMA}_{100}$ (blue squares); emission maximum $\lambda_{\max }$ (filled symbols) and intensity of fluorescence peak (open symbols) of $4 \mathrm{HP}$ as a probe (conditions as in Fig. 1); dark grey circles: data for $\mathrm{PEO}_{114}-b-\mathrm{PPO}_{69}$ for comparison (taken from Fig. 1); part of data is taken from ref. 20 (reprinted with permission from ACS Macro Lett., 2012, 1, 504. Copyright 2012 American Chemical Society).

Above this range, the fluorescence behavior is very much similar to the one of $\mathrm{PEO}_{114}-b-\mathrm{PPO}_{69}$, since the PPO segregation dominates and spherical micelles with a PPO core-domain and PDMAEMA corona are formed.

Compared to similar polymers in the literature, the onset temperature of hydrophobization of $\mathrm{PPO}_{69}-b$-PDMAEMA ${ }_{100}$ is slightly lower. ${ }^{39}$ Partly, this might be again a consequence of the butyl end group, the rather dispersed PPO precursor and the increased salt concentration. Further, the fluorescence intensity shows a maximum in our case, whose reasons can be speculated on. Probably, the combination of PPO and 
PDMAEMA leads to this effect. Since the intensity behavior turned out to be more and more complicated (and sometimes less reproducible) for the following examples, we omit the fluorescence intensity of $4 \mathrm{HP}$ for further discussion and concentrate on the polarity indicator $\lambda_{\max }$. Worthwhile to mention, $\lambda_{\text {max }}$ does not change after entering the two-phase region of PDMAEMA for PPO- $b$-PDMAEMA (system turns turbid). Apparently, the dye prefers to stay in the pure PPO domain, which is hardly influenced by the collapse of the surrounding PDMAEMA. This is in contrast to the results obtained for the miktoarm stars (see below), most probably due to the constraints of the micelle.

In contrast to the block copolymers, solutions of the ternary miktoarm star behave quite differently (see Fig. 3). Both pyrene and 4HP are embedded in a non-polar environment even at low temperatures, though PPO in the diblock copolymers is well hydrated under the same conditions. Most strikingly, the pyrene data strongly suggest a changed microenvironment within the hydrophobic domain when taking into account the lower $I_{1} / I_{3}$ values compared to the ones in pure, non-aqueous PPO as a reference for an ideal hydrophobic PPO domain (refer to the left side of Fig. 3; between 0 and $15{ }^{\circ} \mathrm{C}$ ). This is especially true when considering the ability of tertiary amines to quench the fluorescence of pyrene. ${ }^{45}$ Then still a sufficient fluorescence signal originates from a hydrophobic domain, which has considerably different properties than pure PPO. This indicates a mixed environment for the miktoarm star. This mixed environment can be formed by a complex of PPO and PDMAEMA, which leads to different uptake abilities of the dyes. The complexation renders the PPO hydrophobic within the miktoarm star at basically all temperatures. Thus, complexation occurs even at temperatures below the immanent hydrophobization of the PPO homopolymer. At higher temperatures, the polarity indicators $I_{1} / I_{3}$ or $\lambda_{\max }$ are in the range of $I_{1} / I_{3}$ (or $\lambda_{\max }$ ) seen for PEO- $b$-PPO solutions, though the observed differences between both samples indicate different microenvironments even at elevated temperatures (see below). This clear evidence of hydrophobic compartmentalization at low temperatures is not induced by the presence of PDMAEMA alone. Even combinations (by covalent linkages) of either PPO-PDMAEMA or PEO-PDMAEMA do not lead to a pronounced hydrophobization (as seen in Fig. 2 for $\mathrm{PPO}_{69}-b$-PDMAEMA ${ }_{100}$ and in Fig. 3 for $\left.\mathrm{PEO}_{114}-\left(\mathrm{PDMAEMA}_{110}\right)_{2.5}\right)$. Further, interaction of PEO and PDMAEMA at higher segment densities of PDMAEMA around PEO can be excluded as a reason for the hydrophobicity at low temperatures (as seen for the miktoarm star without PPO in Fig. 3). Only higher segment densities of PDMAEMA around PPO leads to the observed hydrophobic compartmentalization, as seen by the ternary miktoarm star in Fig. 3. When regarding mutual interactions, the driving force for complexation is rather weak, as manifested for $\mathrm{PPO}_{69}-b$-PDMAEMA ${ }_{100}$. However, the complexation within the star is a cooperative process, probably due to the higher number of mutual contacts. In total, this leads to a stronger complex with clear evidence of a changed microenvironment.

The minimum in the $4 \mathrm{HP}$ fluorescence $\lambda_{\max }$ data for $\mathrm{PPO}_{69^{-}}$ $b$-PDMAEMA ${ }_{100}$ and its comparison with PEO $_{114}\left(\text { PDMAEMA }_{90}\right)_{3.1^{-}}$ $\mathrm{PPO}_{69}$ suggest an intermediate special interaction between PPO and PDMAEMA already in the diblock solutions (compare Fig. 2 and 3). In a limited temperature window, the collapsed PPO, which has not aggregated further into micelles, interacts with the PDMAEMA (similar to a zipper by backfolding). Thus, a hydrophobic, unimolecular complex is formed, which is more hydrophobic for the dye than $\mathrm{PEO}_{114}-b-\mathrm{PPO}_{69}$. In contrast to the miktoarm star (see below), the complexation with PDMAEMA occurs only after the hydrophobization of PPO (which takes place at almost the same temperature as for $\left.\mathrm{PEO}_{114}-b-\mathrm{PPO}_{69}\right)$. Further, the weak complex vanishes upon release of water-soluble PDMAEMA at higher temperatures. Obviously, the PDMAEMAPPO complex alone does not induce intermolecular aggregation, which is in line with the low interfacial tension between complex and water. ${ }^{20}$ Apparently, intermolecular aggregation is coupled
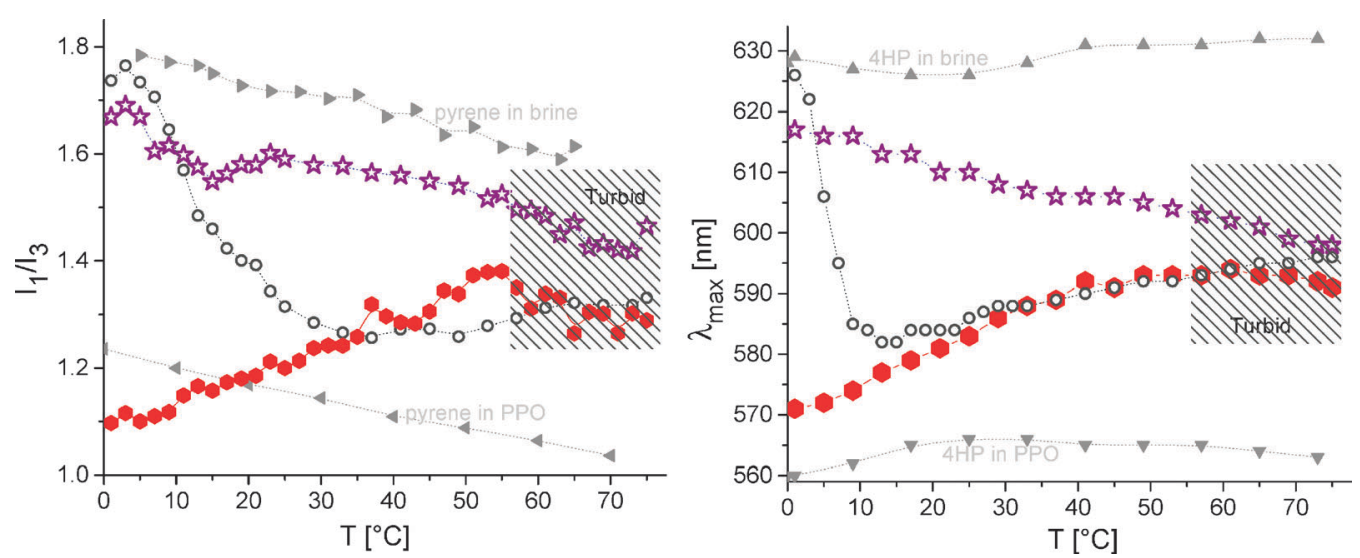

Fig. 3 Fluorescence spectroscopy of different probes in $\mathrm{pH} 8$ buffer $(+0.1 \mathrm{M} \mathrm{NaCl}$ ); left hand side: intensity ratio of the first and third vibronic fluorescence bands of pyrene (conditions as in Fig. 1); right hand side: emission maximum $\lambda_{\max }$ of $4 \mathrm{HP}$ as a probe; assignment: $1.0 \mathrm{~g} \mathrm{~L}^{-1}$ $\mathrm{PEO}_{114}$-(PDMAEMA 90$)_{3.1}-\mathrm{PPO}_{69}$ (red, closed hexagons), $1.0 \mathrm{~g} \mathrm{~L}^{-1} \mathrm{PEO}_{114}-\left(\mathrm{PDMAEMA}_{110}\right)_{2.5}$ (purple, open stars), ${ }^{32}$ for comparison: $0.16 \mathrm{~g} \mathrm{~L}{ }^{-1} \mathrm{PEO}_{114}-b$ $\mathrm{PPO}_{69}$ (dark gray, hollow circles); part of data is taken from ref. 20 (reprinted with permission from ACS Macro Lett., 2012, 1, 504. Copyright 2012 American Chemical Society). 
with a weakening of the complex and PPO segregation for the diblock copolymer at increasing temperatures. Here, the segregating tendency of PPO is stronger than its complexing ability with PDMAEMA (at higher temperatures). The opposite is true for the miktoarm star, where the higher segment densities favor the complexation in contrast to segregation at basically all temperatures.

In addition, the fluorescence data for the PPO-based miktoarm star show a kink in the $I_{1} / I_{3}$ ratio close to the two-phase region of PDMAEMA. This implies that even more PDMAEMA units contribute to the PPO complexation upon PDMAEMA collapse, modifying the hydrophobic microenvironment even further (mixed PPO and PDMAEMA). For the PPO- $b$-PDMAEMA diblock, this effect is not seen, which can be explained by the constraints of the spherical micelle, which prevents a strong interaction between PPO and PDMAEMA. These conclusions are well in line with the IR-results as seen below (Fig. 4).

Regarding $4 \mathrm{HP}, \lambda_{\max }$ is still between the two extremes (pure solvent and pure PPO) at all temperatures, but also here a more detailed analysis reveals microscopic differences in the polarity of the hydrophobic domains both for pure PPO (as seen in the diblock copolymers with collapsed PPO) and the miktoarm star. Therefore, thermodynamic data of the fluorescent dye uptake into the hydrophobic environment were extracted, as already highlighted in our former communication. ${ }^{20}$ It was found that the thermodynamic driving force for dye uptake is very much identical to the two diblock copolymers. However, the miktoarm star showed different enthalpy and entropy of dye uptake, indicating a different microenvironment, which is a result of the complexation of PPO and PDMAEMA. For the miktoarm star, the linear (almost flat) regime of the $\lambda_{\max }$ data of $4 \mathrm{HP}$ at high temperatures, which starts already some degrees before the appearance of turbidity, is most likely a consequence of the PDMAEMA collapse, further increasing the interactions between PPO and PDMAEMA within the miktoarm star and changing therefore the thermodynamics of dye uptake (as was also seen for the pyrene probe). Therefore, both pyrene and $4 \mathrm{HP}$ show nicely the microscopic changes within the miktoarm star at around $50{ }^{\circ} \mathrm{C}$, where further complexation is favored, coinciding with a gradual loss of solubility of PDMAEMA close to its cloudpoint. Interestingly, the PPO-domain of $\mathrm{PPO}_{69}-b$-PDMAEMA micelles hardly interacts with the collapsing PDMAEMA, which is again a consequence of the segregation between the core and the corona of the star-shaped, spherical micelles. Again, the miktoarm star allows special interaction by the close contact between the constituents near the core (see Discussion).

Finally, we turn shortly back to Fig. 3 (also compare Fig. 2). Interestingly, the phase transition of PDMAEMA within a binary miktoarm star of PEO and PDMAEMA does not really change the polarity indicator $\lambda_{\max }$ or $I_{1} / I_{3}$, though PDMAEMA becomes waterinsoluble (see Fig. 3). This is in accordance with the behavior of other PDMAEMA-based systems, as presented in the Introduction. Apparently, the dyes do not see any polarity change of pure PDMAEMA upon phase transition. Similar experiments with e.g. PNIPAM lead to distinct changes in the polarity. ${ }^{15,46,47}$ This implies that PDMAEMA might have a different mechanism of phase separation. Further experiments are required to elucidate this peculiarity within the class of thermoresponsive polymers.

Summarizing the fluorescence part, the complexation within the miktoarm star is detectable throughout the accessible temperature region, though the amount of complexing PDMAEMA increases after its phase transition. The connectivity of PDMAEMA and PPO alone does not lead to any pronounced synergetic induction of hydrophobicity in terms of complexation between the two components. Moreover, the polymeric architecture seems to play a crucial role in this induction of hydrophobicity. The investigated miktoarm star has an increased segment density of PDMAEMA around the PPO arm allowing the favorable contact between the two thermosensitive components (see also Discussion).

\section{IR-spectroscopy}

Temperature-dependent infrared spectroscopy (FTIR) of the carbonyl stretching vibration of PDMAEMA was performed. ${ }^{20}$
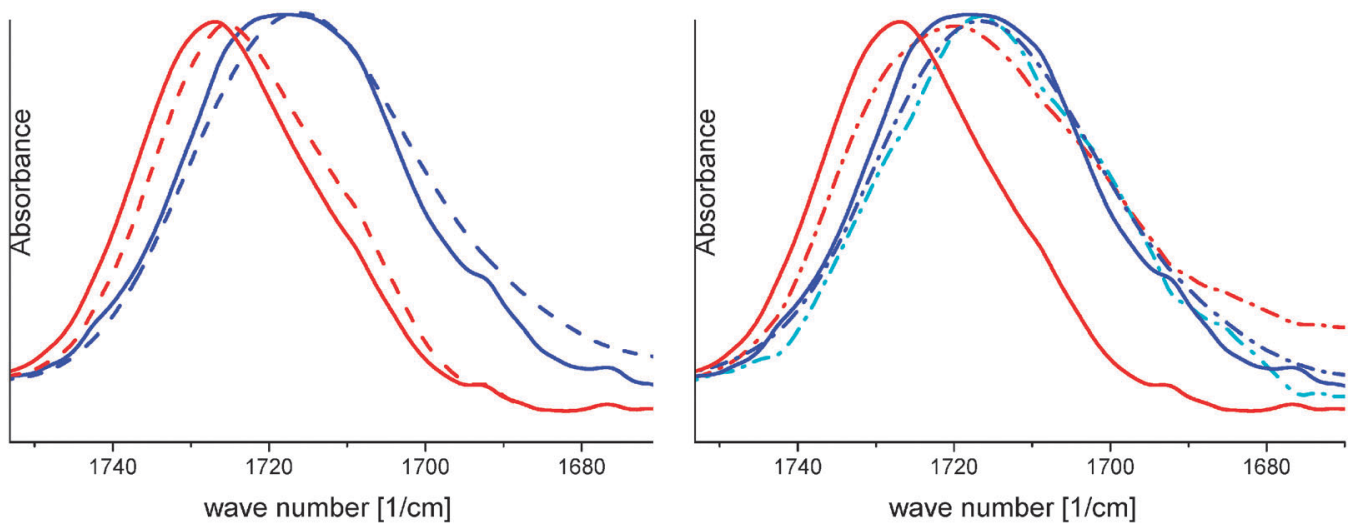

Fig. 4 IR-spectroscopy of the carbonyl band of PDMAEMA; full lines: $\mathrm{PEO}_{114}-\left(\mathrm{PDMAEMA}_{90}\right)_{3.1}-\mathrm{PPO}_{69}$; dashed lines: PEO ${ }_{114}-\left(\mathrm{PDMAEMA} \mathrm{A}_{110}\right)_{2.5}($ (left hand side); dashed-dotted lines: $\mathrm{PPO}_{69}-b$-PDMAEMA ${ }_{100}$ (right hand side); red curves: spectra at $65^{\circ} \mathrm{C}$; blue curves: spectra at $18{ }^{\circ} \mathrm{C}$; cyan curve: spectrum at $3{ }^{\circ} \mathrm{C}$; background subtracted spectra acquisition was performed in $\mathrm{D}_{2} \mathrm{O}$ containing pH 8 buffer, $0.1 \mathrm{M} \mathrm{NaCl}$ and a polymer concentration of $3.0 \mathrm{~g} \mathrm{~L}{ }^{-1}$ $\left(\mathrm{CaF}_{2}\right.$ windows with $0.02 \mathrm{~mm}$ Teflon spacer); part of data is taken from ref. 20 (reprinted with permission from ACS Macro Lett., 2012, 1, 504. Copyright 2012 American Chemical Society). 
Here, we use the IR carbonyl band of PDMAEMA as a probe for its environment (Fig. 4). ${ }^{48}$ Compared to the samples for the fluorescence spectroscopy, the concentration was slightly increased from 1 to $3 \mathrm{~g} \mathrm{~L}^{-1}$ in order to get a reasonable signal. It is observed that the band becomes narrower and shifts to higher wave numbers with increasing temperature. Therefore, the vibronic band of the carbonyl moieties appears at higher wave numbers in a non-aqueous environment. At constant temperature (i.e. comparing the lines of the same color in Fig. 4), the maxima of the PPO-containing miktoarm stars are at higher wave numbers compared to the PPO-free system $\mathrm{PEO}_{114}-\left(\mathrm{PDMAEMA}_{110}\right)_{2.5}$ (which is a good reference). This indicates that the PDMAEMA senses the hydrophobic PPO, both at low and high temperatures. This is well in line with the observation that PPO and PDMAEMA are miscible polymers in the bulk and obviously also in the collapsed state in aqueous dispersion (as seen for the miktoarm star). ${ }^{41}$ Most strikingly, the spectrum of $\mathrm{PEO}_{114}-\left(\mathrm{PDMAEMA}_{90}\right)_{3.1}-\mathrm{PPO}_{69}$ shows a shoulder at higher wave numbers $\left(\sim 1723 \mathrm{~cm}^{-1}\right)$ and at low temperature. This is an indication that some part of the PDMAEMA chain interacts with the PPO even at low temperature, facilitating the hydrophobic compartmentalization of PPO. Again, these favorable van der Waals interactions could be one reason for the miscibility of both polymers in the bulk, where hydrogen bonding and electrostatic interactions are negligible (as PPO and PDMAEMA lack charged sites and possible hydrogen bonding sites). ${ }^{41}$ Obviously, a higher density of neighboring PPO and PDMAEMA units is required, since the diblock $\mathrm{PPO}_{69}-b$-PDMAEMA 100 does not show this behavior at low temperature. Interestingly, the diblock $\mathrm{PPO}_{69}-b$ PDMAEMA $_{100}$ shows an unexpected behavior at elevated temperatures with a broad, shouldered peak. The left-hand slope is again due to collapsed PDMAEMA, though hardly any interaction with hydrophobic PPO can be detected: the left-hand slope of the red dashed-dotted line $\left(\mathrm{PPO}_{69}-b\right.$-PDMAEMA 100$)$ overlaps with the left side of the red dashed peak of the PPOfree miktoarm star $\mathrm{PEO}_{114}-\left(\mathrm{PDMAEMA}_{110}\right)_{2.5}$ but is off the left slope of $\mathrm{PEO}_{114}-\left(\mathrm{PDMAEMA}_{90}\right)_{3.1}-\mathrm{PPO}_{69}$. In contrast, $\mathrm{PPO}_{69}-b$ PDMAEMA $_{100}$ gives a pronounced shoulder on the side of lower wave numbers even at high temperatures, which indicates PDMAEMA in a hydrophilic environment. One possible explanation is given by regarding the constraints of the star-like micelles, which are formed by the hydrophobic PPO. These micelles possess separate domains below the phase-transition of PDMAEMA with a dense hydrophobic PPO core and a "crowded", hydrophilic PDMAEMA corona. In addition, the possible interface per diblock between PPO and PDMAEMA is small in the micelle as well as in the unimer state. Thus, no indication of interaction of PPO and PDMAEMA is found by IR spectroscopy for the diblock at low temperature $\left(3{ }^{\circ} \mathrm{C}\right.$ and $18{ }^{\circ} \mathrm{C}$ ). Interestingly, indications of contact between PPO and PDMAEMA are absent even at elevated temperatures $\left(65{ }^{\circ} \mathrm{C}\right)$. Probably, the outer corona collapses first, leading to a skin layer, which entraps water. Therefore, contact or even mixing of collapsed PDMAEMA with the hydrophobic PPO becomes unlikely. These observations are very much in line with the fluorescence spectroscopy results at elevated temperatures (see above). The situation is different for the miktoarm stars, enabling better contact between PPO and PDMAEMA for $\mathrm{PEO}_{114}-\left(\mathrm{PDMAEMA}_{90}\right)_{3.1}-\mathrm{PPO}_{69}$. In addition, the formation of a skin layer is prevented since the PDMAEMA chains of the star unimers complex with PPO before further aggregation occurs (see below). Including ${ }^{1} \mathrm{H} 2 \mathrm{D}$ NMR NOESY spectra (see ESI $\dagger$ ), there is enough evidence on the attractive interactions between PPO and PDMAEMA even in solution, which lead to complexation as long as the different segments are in close proximity (locked up in a "cozy" microenvironment). In addition, the NMR experiments give first indications of the mechanism of this complexation (see also ESI $\dagger$ ). Here, $m r$ triads of the PDMAEMA backbone provide proximity to the PPO backbone: the $m r$ triads could offer pockets in the right dimensions for the insertion of the PPO backbone, while $r r$ triads are less effective. More in-depth investigations related to the role of tacticity are on the way.

\section{Scattering}

Up to now, we have only learned about the special interactions of PPO and PDMAEMA in the star-shaped geometry. We performed a combined static and dynamic light scattering in order to study the morphology of the polymer aggregates and their structural changes. ${ }^{20,49,50}$ To start with, the $\mathrm{PEO}_{114}-b-\mathrm{PPO}_{69}$ diblock was investigated first (see Fig. 5). Dynamic light scattering revealed the presence of fast-diffusing species (unimers; $\left.R_{\mathrm{h}} \approx 5 \mathrm{~nm}\right)$ up to $17{ }^{\circ} \mathrm{C}$, whereas the first micellar-like structures $\left(R_{\mathrm{h}} \approx 21 \mathrm{~nm}\right)$ appeared already at $5{ }^{\circ} \mathrm{C}$. At all temperatures, a slow-diffusive process $\left(R_{\mathrm{h}} \approx 75-150 \mathrm{~nm}\right)$ was detected. This unexpected slow mode has also been observed for other PEO/PPO-based and for many other water-soluble polymers and can be regarded as "loose" aggregates. ${ }^{46,51-54}$ Even pure PEO shows such an "associative" aggregation in aqueous solution. ${ }^{55}$ Interestingly, the overall scattering intensity exhibits a maximum for $\mathrm{PEO}_{114}-b-\mathrm{PPO}_{69}$ upon heating between 9 and $29{ }^{\circ} \mathrm{C}$, which can be undoubtedly assigned to non-specified rearrangements of the loose aggregates $\left(R_{\mathrm{g}}\right.$ rather constant at $\sim 150 \mathrm{~nm}$; Fig. 5 ). At the same time, the $R_{\mathrm{g}} / R_{\mathrm{h}}$ value has a pronounced kink at $20{ }^{\circ} \mathrm{C}\left(R_{\mathrm{g}} / R_{\mathrm{h}}\right.$ shifts from approximately 1.0 to 1.6 ), indicating again some rearrangements of the loose aggregates into structures, which are probably more drained by solvent. Then, the aggregation number and/or the number of (spherical) micelles increases between 13 and $25{ }^{\circ} \mathrm{C}$, as was expected for these thermoresponsive polymers. The onset of this population change coincides well with the onset of the turbidity curve of pure $\mathrm{PPO}_{69}$ (cloud point $T_{\mathrm{cp}}=13{ }^{\circ} \mathrm{C}$ of a $0.05 \mathrm{~g} \mathrm{~L}^{-1}$ aqueous solution in $\mathrm{pH} 8$ buffer and $0.1 \mathrm{M}$ brine), ${ }^{24}$ though hydrophobic domains were found already above $5{ }^{\circ} \mathrm{C}$. Thus, PPO starts to dehydrate partially even before its cloud point, as was seen for other thermosensitive polymers. ${ }^{27,56}$ In turn, the assignment of the two-step micellization is reasonable, as was done during the interpretation of the fluorescence results (see Fig. 1). Hysteresis during the heating and cooling cycles is discussed in the ESI. $\dagger$ In summary, the combination of 

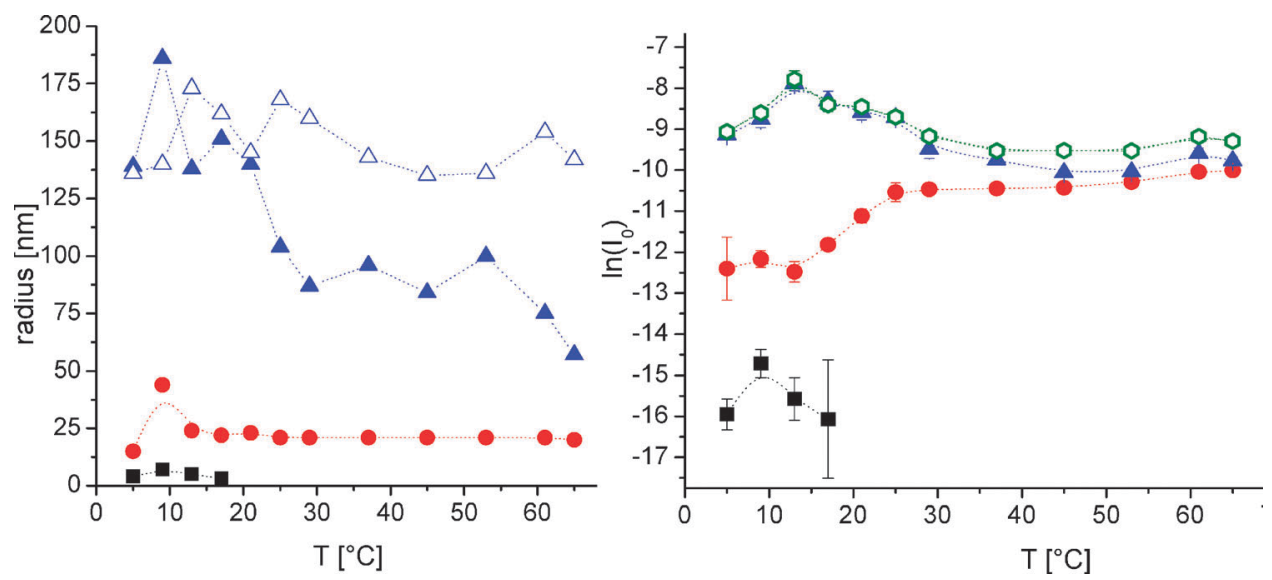

Fig. 5 Combined DLS (CONTIN) and SLS results of $0.16 \mathrm{~g} \mathrm{~L}^{-1} \mathrm{PEO}_{114}-b-\mathrm{PPO}_{69}$ in $\mathrm{pH} 8$ buffer and $0.1 \mathrm{M} \mathrm{NaCl}$; left hand side: hydrodynamic radii of small- (squares), medium- (circles) and large-sized (triangles) species as seen by CONTIN analysis (full symbols; extracted for each species from the slope of decay rate against $q^{2}$ ) and radius of gyration of largest species (open triangle; extracted by Guinier plot after a decomposing combination of angular DLS and SLS treatment as used for the right hand side data); right hand side: primary combined intensities (small, open and green hexagons) together with their split intensities of each species by a combined DLS and SLS treatment (same symbols as on the left hand side; all intensities obtained after extrapolation $q \rightarrow 0$; heating curves; lines are guide to the eye); part of data is taken from ref. 20 (reprinted with permission from ACS Macro Lett., 2012, 1 , 504. Copyright 2012 American Chemical Society).

fluorescence spectroscopy and light scattering reveals some hidden features of the self-assembly process of $\mathrm{PEO}_{114}-b-\mathrm{PPO}_{69}$.

Therefore, we used the same approach also for the other diblock copolymer $\left(\mathrm{PPO}_{69}-b\right.$-PDMAEMA 100$)$, which was helpful for comparison. Loose aggregates are found throughout the temperature scan $\left(R_{\mathrm{h}} \approx 110 \mathrm{~nm} ; R_{\mathrm{g}} / R_{\mathrm{h}}\right.$ changes from approximately 1 to 1.3 between 10 and $20{ }^{\circ} \mathrm{C}$ ), until further aggregation takes place upon entering the two-phase LCST region of PDMAEMA at approximately $58{ }^{\circ} \mathrm{C}$. In contrast to $\mathrm{PEO}_{114^{-}} b$ $\mathrm{PPO}_{69}$ solution, the intensity of the loose aggregates does not show a maximum at low temperatures, but the number/molecular weight of the loose aggregates increases upon heating the sample from 1 to $17^{\circ} \mathrm{C}$.

Irrespective of the behavior of the loose aggregates, both diblocks show a rather similar self-assembly pattern as seen in the light scattering analysis. Again, the combined DLS and SLS evaluation of a $\mathrm{PPO}_{69}-b$-PDMAEMA 100 solution detects unimers up to $13{ }^{\circ} \mathrm{C}\left(R_{\mathrm{h}} \approx 5 \mathrm{~nm}\right)$, whereas the first micelles appear at $5{ }^{\circ} \mathrm{C}\left(R_{\mathrm{h}} \approx 18 \mathrm{~nm}\right.$; see Fig. 6). The number of micelles increases especially between 10 and $20^{\circ} \mathrm{C}$. At approximately $58^{\circ} \mathrm{C}$, further aggregation takes place upon entering the two-phase LCST region of PDMAEMA.

Finally, the combined DLS and SLS evaluation of a $\mathrm{PEO}_{114^{-}}$ $\left(\mathrm{PDMAEMA}_{90}\right)_{3.1}-\mathrm{PPO}_{69}$ solution detects the presence of different species up to the onset of turbidity, which was induced by the aggregation of PDMAEMA at $55{ }^{\circ} \mathrm{C}$ (Fig. 7). The loose aggregates, which are also present in the miktoarm stars, do not show any structural rearrangements upon heating $\left(R_{\mathrm{h}} \approx\right.$ $\left.80 \mathrm{~nm} ; R_{\mathrm{g}} / R_{\mathrm{h}}=1.6\right)$, until successive aggregation takes place by entering the two-phase LCST region of PDMAEMA. This corroborates the already collapsed structure of PPO at low temperature. In addition, the temperature dependence of the respective intensities does not show pronounced changes. Upon heating, a moderate increase in the number of unimers and micelles
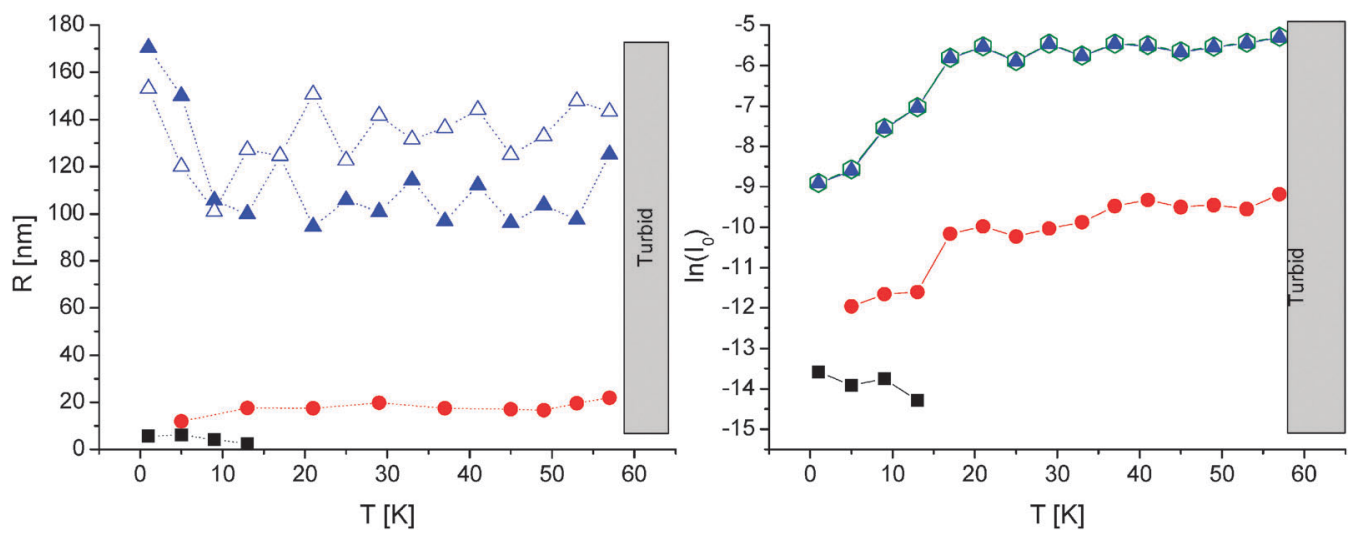

Fig. 6 Combined DLS (CONTIN) and SLS results of $0.4 \mathrm{~g} \mathrm{~L}^{-1} \mathrm{PPO}_{69}-b-\mathrm{PDMAEMA}_{100}$ in $\mathrm{pH} 8$ buffer and $0.1 \mathrm{M} \mathrm{NaCl}$; otherwise, the assignment is the same as in Fig. 5; part of data is taken from ref. 20 (reprinted with permission from ACS Macro Lett., 2012, 1, 504. Copyright 2012 American Chemical Society). 

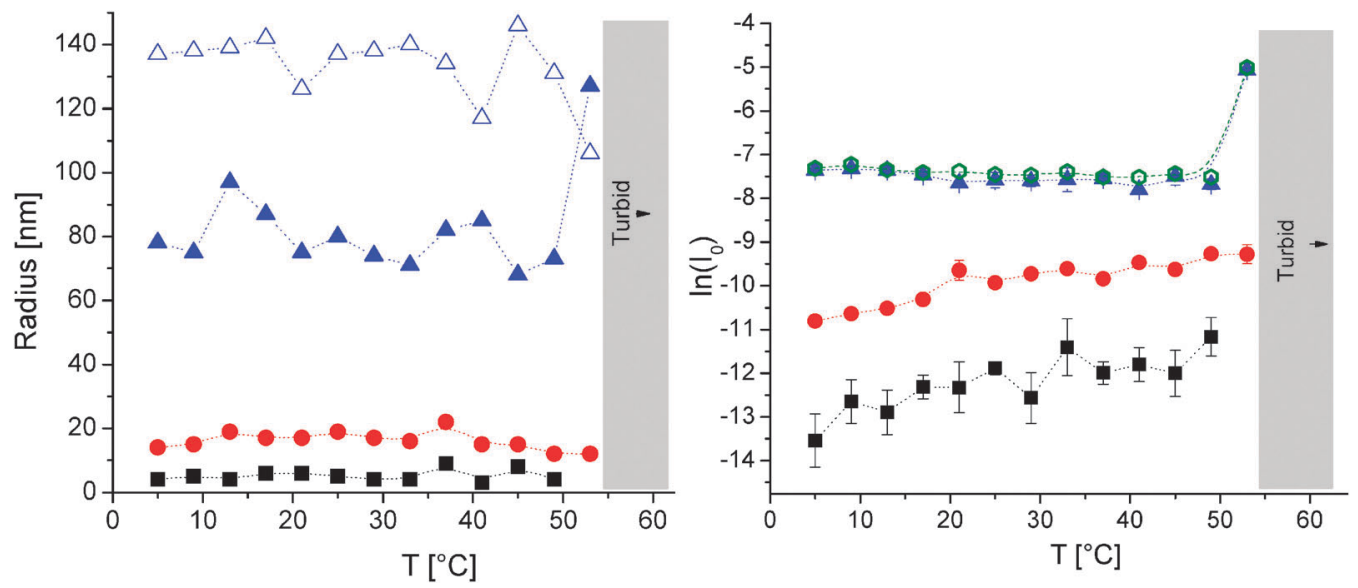

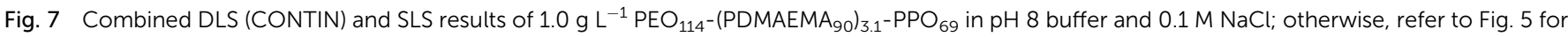
further assignment; part of data taken from ref. 20 (reprinted with permission from ACS Macro Lett., 2012, 1, 504. Copyright 2012 American Chemical Society).

seems to take place, which is accompanied by a slight decrease in the intensity of the loose aggregates. In contrast to the diblocks, star unimers were seen up to $50{ }^{\circ} \mathrm{C}\left(R_{\mathrm{h}} \approx 5 \mathrm{~nm}\right)$ as well as micelles, which appeared from $1{ }^{\circ} \mathrm{C}$ to $53{ }^{\circ} \mathrm{C}\left(R_{\mathrm{h}} \approx\right.$ $16 \mathrm{~nm})$. It is worthy of note that the star unimers have almost the same hydrodynamic radius as was observed for $\mathrm{PEO}_{114^{-}} b$ $\mathrm{PPO}_{69}$. This is well in line with a partly collapsed structure of the miktoarm stars in the form of unimolecular micelles. Apparently, the size of these unimolecular micelles does hardly change with temperature, whereas there might be a slight decreasing trend in size of the diblock unimers (collapse of $\mathrm{PPO}$ ). Compared to the parental $\mathrm{PEO}_{114}-b-\mathrm{PPO}_{69}$, the micellartype aggregates of the stars have a considerably smaller $R_{\mathrm{h}}$ ( $R_{\mathrm{h}} \approx 16 \mathrm{~nm}$ compared to $R_{\mathrm{h}} \approx 21 \mathrm{~nm}$ ). This is in accordance with a rather low number of aggregation, which is probably the result of decreased interfacial tension due to complexation and/ or the crowding of the mixed PDMAEMA-PEO-corona due to the branched structure of the miktoarm star. Additionally, the micellar aggregates are likely to be composed predominantly of miktoarm stars with lower arm numbers, since the preparation of the miktoarm stars leads to a PDMAEMA arm number distribution with a number average of $3.1 \mathrm{arms}$ per molecule (see also Table 1).$^{42}$ Most strikingly, the difference in the order of magnitude between the scattering intensity of unimers and micelles is rather small as compared to the diblocks. This is another indication that the aggregation number of the micelles is rather small and/or the concentration of miktoarm micelles is reduced compared to the unimolecularly dissolved polymer. This is especially true when taking into account the non-linear scaling of the scattering intensity with molar mass (and size). As shown earlier, ${ }^{20}$ we can use a rough semi-quantitative approach in order to estimate the mass concentration of each scattering entity by using the Daoud and Cotton theory for starshaped objects like polymer stars or star-shaped micelles. ${ }^{57}$ The theory correlates the size of the object $R$ with the aggregation number $N_{\text {agg }}$ (by assuming a constant arm length). This theory is strictly valid only in an intermediate regime of $N_{\text {agg }}$ and has its limitations for the unimers $\left(N_{\text {agg }}=1\right)$ and especially for the loose aggregates. A combination of the Zimm treatment and the Daoud and Cotton theory allows the extraction of the mass concentration $c$ of each species by its scattering intensity according to the following formula:

$$
c \sim I / R^{5}
$$

The results are shown in Fig. $8 .{ }^{20}$ At low temperature, the dominant species are the unimers for both $\mathrm{PEO}_{114}-b-\mathrm{PPO}_{69}$ and $\mathrm{PEO}_{114}-\left(\mathrm{PDMAEMA}_{90}\right)_{3.1}-\mathrm{PPO}_{69}$. In contrast, the diblock has self-assembled predominantly into micelles at elevated temperature, whereas hardly any aggregation occurs for the miktoarm star.

For further comparison, we measured SAXS (small angle X-ray scattering) of a $\mathrm{PEO}_{114}\left(\mathrm{PDMAEMA}_{90}\right)_{3.1}-\mathrm{PPO}_{69}$ solution and a $\mathrm{PEO}_{114}-b-\mathrm{PPO}_{69}$ solution at low and high temperatures (ESI $\dagger$ ). As a result, the aggregation number $N_{\text {agg }}$ of $\mathrm{PEO}_{114^{-}}$ $b-\mathrm{PPO}_{69}$ was estimated to be in the order of 200 at high temperature,$^{58}$ which is consistent with former investigations on similar micellar systems. ${ }^{59}$ Here, $N_{\text {agg }}$ is about 10 times larger than the values obtained by fluorescence quenching experiments (see ref. 20; this discrepancy can be easily explained by the 6 times larger hydrophobic core volume compared to the Förster volume ${ }^{60}$ in the case of unimolecular micelles, the Förster volume is larger than the hydrophobic domain size and $N_{\text {agg }} \sim 1$ obtained by fluorescence quenching is realistic). Further, we also measured SAXS of the miktoarm star solution, though we could not access a broader scattering range. Thus, only the Guinier regimes were recorded (ESI $\dagger$ ): the data on the radii of gyration corroborate again the internal compartmentalization. Noteworthily, the ratios of the extrapolated intensities $I_{0}$ for the miktoarm star unimers and miktoarm star micelles observed by the DLS/SLS approach and by the SAXS measurements coincide well. This is another indication that the decomposing light scattering treatment works well and gives reasonable results. 

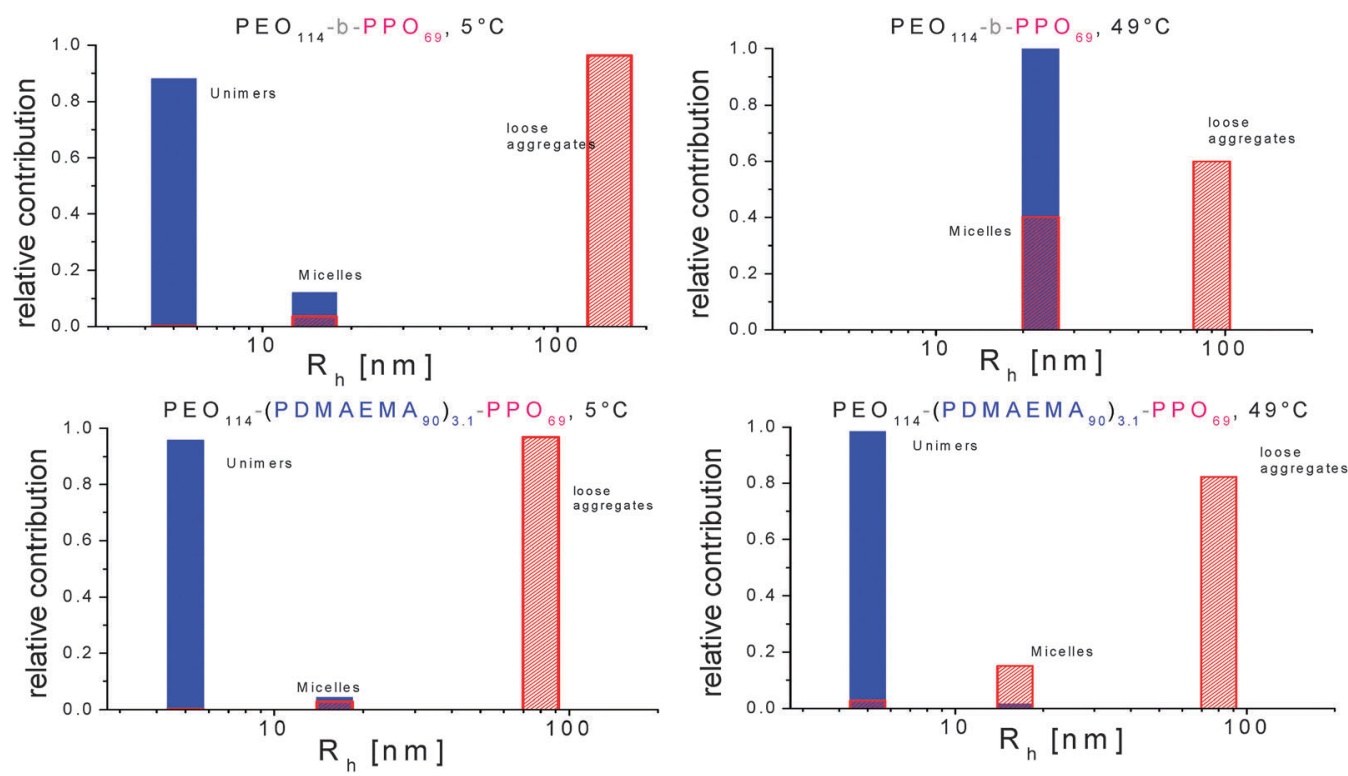

Fig. 8 Comparison of intensity (extrapolated to $q \rightarrow 0$; red, open striped bars) with the mass concentration by use of the Daoud-Cotton scaling (blue full bars); left diagrams are at $5{ }^{\circ} \mathrm{C}$, right hand side is obtained at $49{ }^{\circ} \mathrm{C}$; top row: $\mathrm{PEO}_{114}-b-\mathrm{PPO}_{69}$, bottom row: $\mathrm{PEO}_{114}-\left(\mathrm{PDMAEMA}_{90}\right)_{3.1}-\mathrm{PPO}_{69}$; part of data is taken from ref. 20 (reprinted with permission from ACS Macro Lett., 2012, 1, 504. Copyright 2012 American Chemical Society).

We can conclude that the majority of the stars are present as unimolecular micelles. This behavior can be explained by the complexed and retracted PPO together with the reduced interfacial tension (see also Discussion). However, the formation of polymer micelles and polymer aggregates cannot be totally avoided here (as seen by DLS). Again, stars with a lower number of PDMAEMA arms (compared to average 3.1 PDMAEMA arms per molecule) might be the predominant reason for these few micellar structures, as the preparation of the miktoarm star leads to a moderate arm number distribution in PDMAEMA arms. ${ }^{42}$ This allows the intermolecular interaction of the hydrophobic domains for stars with decreasing arm number, whose behavior resembles more and more the behavior of the diblock copolymers. By use of cryogenic Scanning Transmission Electron Microscopy (cryo-STEM; see ESI $\dagger$ ), micellar structures could be detected, which are well in line with the light scattering results.

\section{Unimolecular to multimolecular micelle transition}

So far, we have only addressed the solution properties of the miktoarm star at temperatures below the phase transition of the PDMAEMA chains. Therefore, we present a preliminary study on the higher order aggregation at elevated temperatures. Entering the two-phase region of PDMAEMA, the star solution turns strongly turbid (leading to multiple scattering in DLS). Turbidity indicates either macroscopic phase separation or the presence of colloidally stable, high molar mass aggregates. Most likely, the latter explanation is valid also for our case, as microscopy studies have shown the presence of micrometer sized aggregates, which were stable for days at elevated temperature (Fig. 9).

Besides optical microscopy, Scanning Electron Microscopy of vitrified samples (cryo-SEM) gave further evidence of spherical

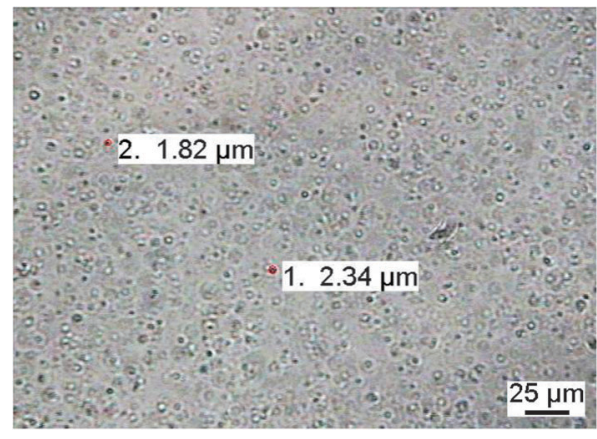

Fig. 9 Optical micrograph of a $1 \mathrm{~g} \mathrm{~L}^{-1}$ solution of $\mathrm{PEO}_{114^{-}}\left(\mathrm{PDMAEMA}_{90}\right)_{3.1^{-}}$ $\mathrm{PPO}_{69}$ (in $\mathrm{pH} 8$ buffer and $0.1 \mathrm{M} \mathrm{NaCl}$ ) at $70{ }^{\circ} \mathrm{C}$.

polymer aggregates in the size range of 1-7 $\mu \mathrm{m}$ in diameter after partial sublimation of water (see ESI $\dagger$ ). Finally, cryo-STEM (Fig. 10) was possible on those spots of the sample, where only a thin layer of vitrified water was seen before (partial sublimation led to some salt crystals, which could be distinguished from the spherical polymer aggregates). Then, the size of the aggregates is smaller than the sizes seen by cryo-SEM, due to the fractionating effect of the thin water layer. However, the cryo-STEM results indicate the absence of only unilamellar vesicular structures and favor the presence of multilamellar vesicles ("onions"). In some cases, the cryo-STEM images give the impression of onion-type structures (with apparent lamellar spacings $\approx 100-150 \mathrm{~nm}$, marked by arrows in Fig. 10, though we could not detect any lamellar layering by SAXS at $70{ }^{\circ} \mathrm{C}$ (missing of structure peaks; data not shown)). This might suggest interlamellar spacings larger than the resolution of our SAXS $(\geq 150 \mathrm{~nm})$ for these self-assembled structures. Therefore, we propose as the most probable structure an onion-type assembly with mixed PPOPDMAEMA membranes, which are water-insoluble and which 


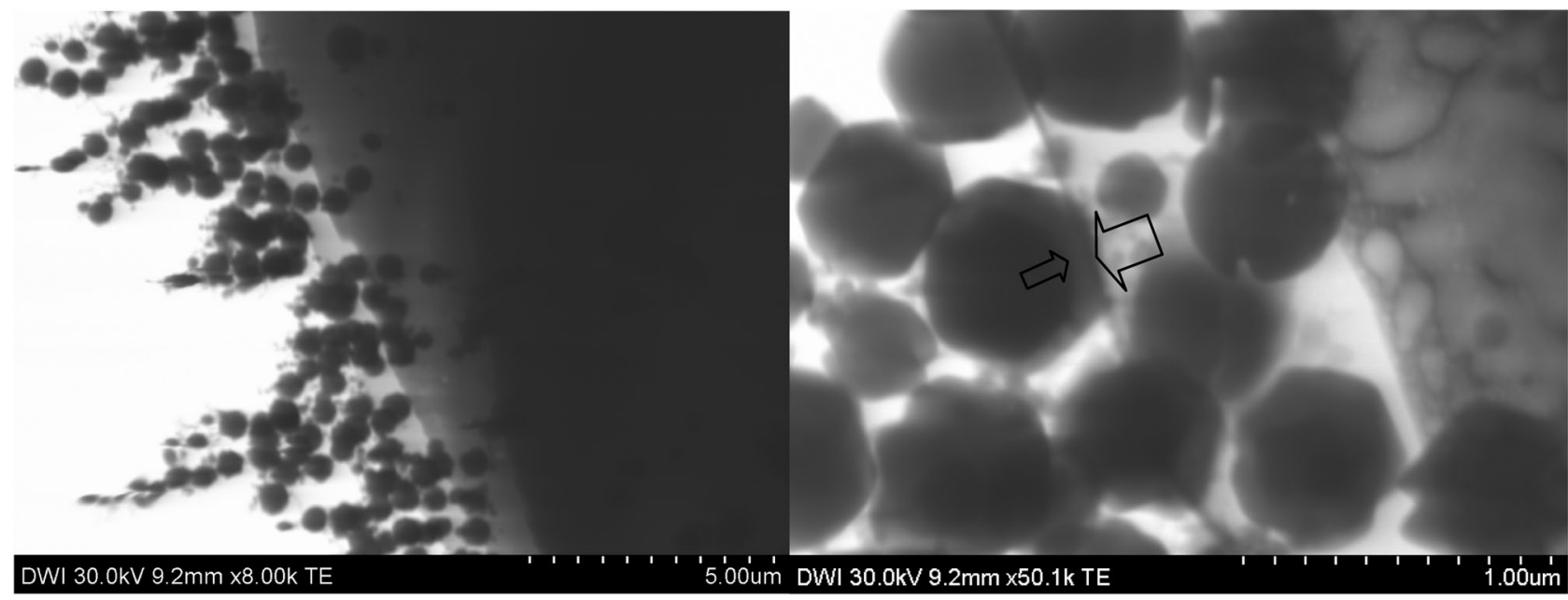

Fig. 10 Cryo-STEM images of $1 \mathrm{~g} \mathrm{~L}^{-1}$ solution of $\mathrm{PEO}_{114}-\left(\mathrm{PDMAEMA}_{90}\right)_{3.1}-\mathrm{PPO}_{69}$ (in $\mathrm{pH} 8$ buffer and $0.1 \mathrm{M} \mathrm{NaCl}$ ) kept at $70{ }^{\circ} \mathrm{C}$ overnight and vitrified from approximately $70^{\circ} \mathrm{C}$ (water has been partially sublimed within the microscope; the appearance of multilamellar vesicles are marked by exemplary arrows; scale bars of 5 and $1 \mu \mathrm{m}$ total size are subdivided into 10 parts as indicated by the white lines).

are sandwiched by a PEO brush. This leads to steric stabilization of the whole multilamellar vesicle as such and of each onion layer as well. Most noteworthy, this transition from unimolecular to multimolecular micelles crosses the widest spectrum of micellar morphologies. Instead of the transitions from unimolecular to star-shaped micelles for the diblock copolymers (which is a transition between neighboring morphologies), the star unimers assemble into multilamellar vesicles without any indications of (intermediate) star-like or worm-like micellar structures. This unprecedented transition is a consequence of two mechanisms both promoted by the star-shaped topology: the internal complexation prevents PPO-triggered aggregation and the star-shaped architecture forces the self-assembly into peculiar vesicular structures upon phase transition of the majority component PDMAEMA. ${ }^{61}$

\section{Discussion}

We first summarize the most striking indications for complexation between PPO and PDMAEMA within the miktoarm star:

(a) The pyrene method shows a lower $I_{1} / I_{3}$ value in the case of the miktoarm star than for pyrene in pure PPO. That means that the hydrophobic compartment appears to be more hydrophobic than pure PPO.

(b) Also the 4HP data indicate a changed microenvironment, which is seen by changes in the thermodynamic parameters of the $4 \mathrm{HP}$ uptake into the hydrophobic domain. ${ }^{20}$

(c) The IR data show that there are PDMAEMA carbonyl groups in a water-insoluble/hydrophobic environment at low temperature for the PPO-based miktoarm star.

(d) The NOESY-NMR data directly suggest spatial proximity of PDMAEMA and PPO within the miktoarm star (ESI $\dagger$ ). In addition, we have also seen that the PPO methyl signal has shifted downfield (by $0.1 \mathrm{ppm}$ ) for the miktoarm star compared to the diblock, which again indicates a different microenvironment for the PPO (as discussed in the ESI $\dagger$ ).

(e) Simple ${ }^{1} \mathrm{H}$-NMR data suggested complexation, as shown in our former publication. ${ }^{20}$ Both the PDMAEMA and PPO signal intensity diminishes considerably upon transferring the miktoarm star from organic solvent into water (while the PEO signal is basically unchanged). Again, this is another indication that there is complex formation between PPO and PDMAEMA, which is more viscous and less hydrated, leading to PPO and PDMAEMA units with decreased mobility and decreased visibility in the NMR experiment.

(f) As discussed below, the prevalence of unimolecular micelles can be only caused by a significant reduction of interfacial tension between PPO domain and bulk water, which can be a result of complexation of the hydrophobic PPO with the more hydrophilic PDMAEMA.

Further, we summarize the structural evolution with temperature for all polymers in Schemes 1 and 2. The most probable micellization scenario of the diblock copolymers is concluded on the basis of the scattering data and of the IR and fluorescence spectroscopy results (see above and Scheme 1). In turn, the behavior of the miktoarm star (Scheme 2) is changed compared to the behavior of the diblock copolymers, which is rich in various structures present at different temperatures (Scheme 1). Within the accessible temperature region, there is only one transition between unimolecular micelles and higher order aggregation (probably multilamellar vesicles). A similar intermolecular aggregation behavior is observed for $\mathrm{PEO}_{114}-\left(\mathrm{PDMAEMA}_{110}\right)_{2.5}$.

As already outlined throughout this paper and in our previous communication, ${ }^{20}$ we have argued that a segment density effect accounts for the induction of complexation/hydrophobicity of PPO within the PDMAEMA-based miktoarm star at low temperature. At this point, an additional effect of the overall molecular weight (including PDMAEMA and PEO branches) on the thermosensitivity of PPO cannot be excluded (e.g. lowering 

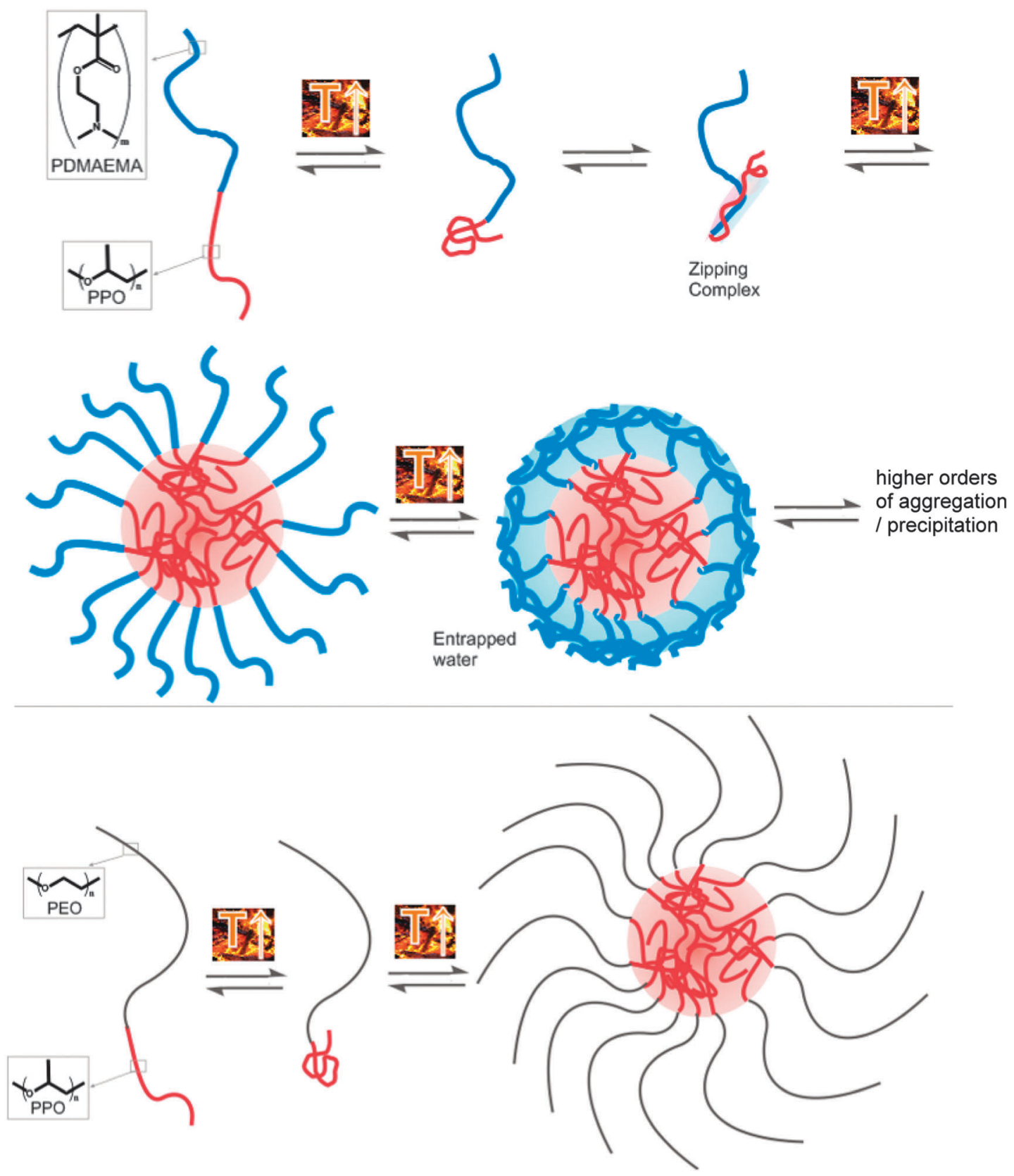

Scheme 1 Solution behavior of diblock copolymers.

the LCST due to increased molecular weight). However, it can be excluded that a possible molecular weight effect is the only reason for the hydrophobization of PPO at low temperatures, since enough evidence of complexation between PPO and PDMAEMA could be found. Thus, topological effects do play a role. Generally, topological effects of star polymers become more pronounced for higher numbers of arms. ${ }^{62}$ E.g. the increase in segment density at the outer rim of the star is rather limited for low arm numbers. Taking the same hydrodynamic radius for both diblock and star unimers ( $5 \mathrm{~nm}$; Fig. 6 and 7), the averaged local segment density of PDMAEMA is only a factor 3 higher within the star compared to its density within the hydrodynamic volume of the diblock unimers $\left(c_{\text {DMAEMA }}=1 \mathrm{M}\right.$ compared to
$0.3 \mathrm{M} ; c_{\mathrm{PO}}=0.2 \mathrm{M}$ for both cases). Is the stated explanation then reasonable with respect to the rather low number of PDMAEMAarms? We think that the local concentration near the core of the star is essential for understanding this behavior. It is almost an order of magnitude higher than the average "intra-star" concentration when taking a hypothetical first monomer layer around the core of the star (first "generation" with a $0.7 \mathrm{~nm}$ radius, as estimated from the formula stated in ref. 20 and 42 and in the ESI; $\dagger$ the formulas together with Scheme 3 would suggest $c_{\text {DMAEMA }}($ inner sphere) $\sim 5 \mathrm{M}$ ). Within this layer, the segment density approaches even the bulk concentration of PDMAEMA (in pure DMAEMA: $c_{\text {DMAEMA }} \sim 6 \mathrm{M}$ ). The segmental effect close to the junction point is less pronounced for diblock copolymers. 

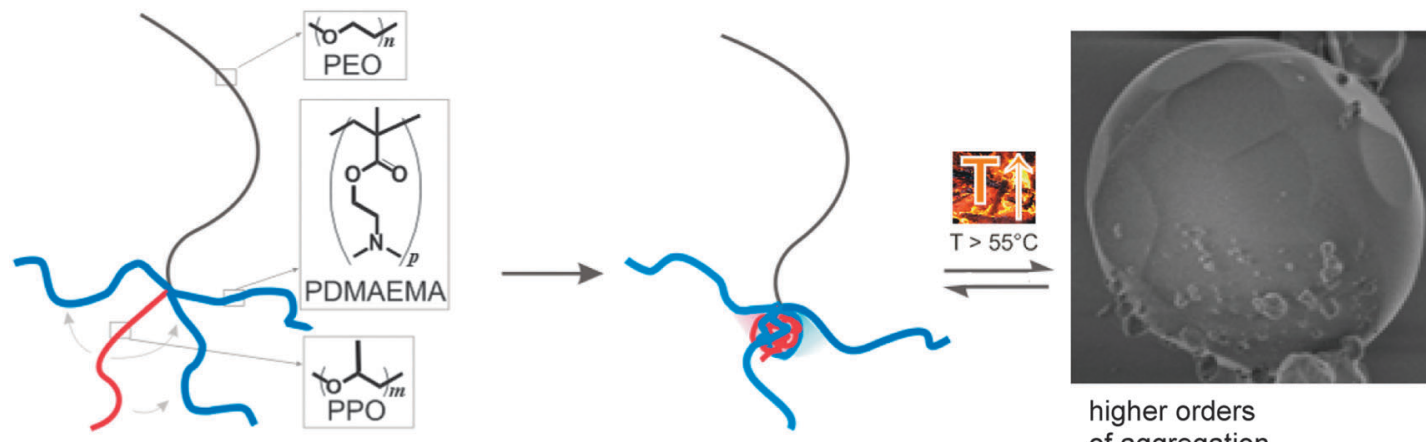

higher orders

of aggregation

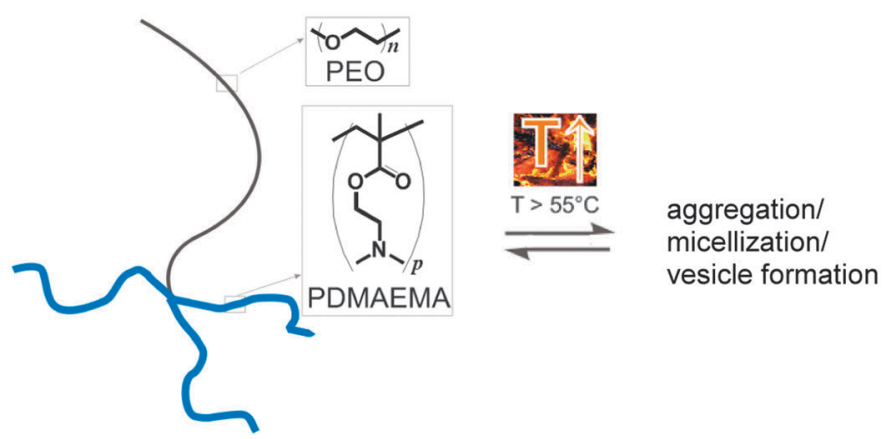

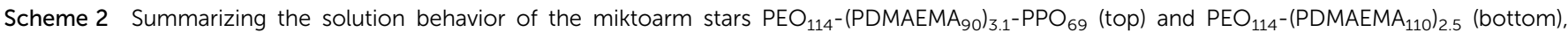
showing the complexation between PPO and PDMAEMA at basically all temperatures (exemplary cryo-SEM micrograph is taken from the ESI $\dagger$ ).
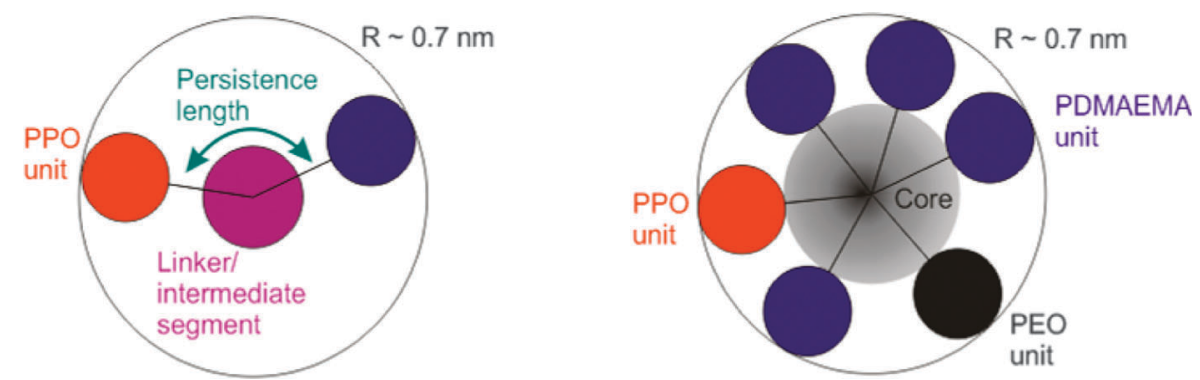

Scheme 3 Simplified segmental effects near the junction point of the blocks (left side: diblock copolymer; right side: miktoarm star).

Also here, the segment density of one constituent around the other constituent is highest close to the junction point. But, the difference to the average segment density is rather small. In addition, the persistence length is typically in the order of the diameter of the first "monomer sphere" (see Scheme 3) ${ }^{63}$ Thus, the persistence length keeps the monomer units, which are adjacent to the junction point, apart, unless one block collapses. In the case of the miktoarm star, the concept of persistence length is rather meaningless on the length scale of the inner "monomer sphere". Therefore, close contact of the PDMAEMA units with the PPO units is an immanent feature of the starshaped system leading locally to a situation similar to the situation in bulk mixtures. ${ }^{41}$ The close contact triggers the weak, but favorable interactions between PPO and PDMAEMA, leading to a hydrophobic domain at the center of the star. Then, this domain can act as a nucleus for further complexation between
PPO and PDMAEMA, as suggested by the IR-data. In order to see the shoulder in the spectrum at low temperature, more contact points between PDMAEMA and PPO are needed as just the few within the inner sphere (first "generation"). Otherwise, detailed information is lacking on the size and on the internal structure of the hydrophobic domain. However, it is likely that not all PDMAEMA arms take part in the complexation (Scheme 2). Probably, some arms interact preferentially with PPO, leaving the other PDMAEMA arms rather unaffected, as was shown for other polyelectrolyte complexes (disproportionation). ${ }^{64}$

It will be interesting to see in the future, whether stars with considerably longer PPO arms (or shorter PDMAEMA arms) will still form unimolecular micelles or would self-assemble intermolecularly. The first scenario would then be again a consequence of the internal complexation, leading to a "towing" of a long PPO arm into the core of the micelle. Still, the contour 
lengths of the PDMAEMA and PPO chains are rather comparable in our case (taking into account that PDMAEMA has only 2 atoms and PPO has 3 atoms in the backbone per repeating unit). This is in contrast to the longer solvophilic chains, which protected the segregated part in other miktoarm stars. ${ }^{19}$

As already pointed out in our previous work, ${ }^{20}$ a scaling approach helped to understand the origin of the prevalence of unimolecular micelles. ${ }^{65}$ These theoretical relationships do not take charging effects into account, though charging would reduce $N_{\text {agg }}$ further (highly unfavorable charge concentration and osmotic pressure within the corona of proposed miktoarm micelles due to increased PDMAEMA segment density). However, negligence of charges is reasonable for our system: only approximately every 5th PDMAEMA unit bears a charge at low temperature (at pH 8; see ESI $\dagger$ ). Further, the electrostatical contributions to unimer stabilization are effectively weakened by the medium ionic strength, which leads to a smaller Debye length than the average charge-to-charge separation along the PDMAEMA chain. This effective screening leads to a rather similar behavior of the uncharged PEO- $b$-PPO and PPO- $b$ PDMAEMA diblock copolymers. Even more, the stars turn basically uncharged at elevated temperatures. ${ }^{30}$ This was seen by titration (please refer to the ESI $\dagger$ ). But also at lower temperatures, the polymer is expected to be rather uncharged close to the core of the star (which might favor again complexation with PPO): due to a release of high local osmotic pressure, an inhomogeneous distribution of charges is expected within stars. ${ }^{66}$ Taking now into account Fig. 8, the amount of aggregated stars did even decrease from 5 to $49{ }^{\circ} \mathrm{C}$, though the amount of charged monomer units decreased considerably. Hence we conclude that electrostatic stabilization does not play a major role for our system. Moreover, the scaling approach revealed that the star architecture alone and the geometrical effects of a possible complexation do not explain the drastic decrease in aggregation number when going from diblock to miktoarm star. If now neither the electrostatic repulsion between the stars nor the enhanced sterical coronal constraints within the proposed miktoarm star micelles is responsible for this unique behavior, what can be the origin of the prevalence of unimolecular micelles? The answer is then the reduction of interfacial tension between the PPO domain and the bulk solvent. As shown before, the presence of (linear) PDMAEMA leads to a substantial decrease in the interfacial tension of (linear) PPO towards water (at elevated polymer concentrations). ${ }^{20}$ This effect, which is based on the complexation between both polymers, is also imitated on smaller length scales within the miktoarm star (PDMAEMA acts like a "microsurfactant"), but the amount of mutual interactions is not sufficient to induce an effective complexation for the diblock copolymers at low concentrations. This was also seen for other interacting polymer species like poly $(N, N$-diethyl acrylamide $)$ and poly( $N$-isopropyl acrylamide). ${ }^{48,67}$ While pronounced complexation (here by hydrogen bonding) was present for statistical copolymers, block copolymers did not show specific interactions between the complexing partners. Again, the spacial proximity between the weakly interacting partners is essential.
In the case of PPO and PDMAEMA, such a statistical copolymerization is very difficult to achieve. Alternatively, a star-like, heteroarm topology can be used to attain a sufficiently high mutual segment density.

\section{Conclusions}

By use of a miktoarm star, we have shown that the local microenvironment has a tremendous influence on the properties of polymer chains in aqueous solution. This is especially true for systems with a weak attractive interaction between the two different homopolymers. Here, a higher segment density of PDMAEMA around one PPO chain is required in order to induce an efficient complexation between PPO and PDMAEMA in water. For the corresponding diblock copolymers, this interaction is hardly detected, since the segmental density of the PDMAEMA around the PPO is low in solution for diblock copolymers. In contrast, the internal complexation within the miktoarm star leads to hydrophobic compartments with lowered interfacial tension towards bulk water. At the same time, non-centrosymmetric (Janus-type) unimolecular micelles are formed under conditions in which all single constituents of the miktoarm star are highly water-soluble. Thus, this article describes two "micro-hierarchical" sequences of microscopic events:

(i) for PPO-PDMAEMA-based miktoarm stars: (intramolecular) complexation $\rightarrow$ hydrophobization and collapse of the complex/lowering of the interfacial tension between the complex and bulk solvent at the same time $\rightarrow$ prevention of aggregation $\rightarrow$ prevalence of unimolecular micelles.

(ii) for PPO- $b$-PDMAEMA diblock copolymers: hydrophobization $\rightarrow$ collapse/backfolding $\rightarrow$ (intramolecular complexation $\rightarrow$ decomplexation) $\rightarrow$ aggregation $\rightarrow$ prevalence of spherical micelles.

Therefore, complex macromolecular architectures can lead to an unprecedented intramolecular self-assembly behavior, where - under certain circumstances - the internal complexation prevents further intermolecular self-assembly upon the formation of unimolecular micelles.

The results of Monte Carlo simulations, which support the influence of polymer architecture on complex formation, will be presented in a subsequent publication.

\section{Acknowledgements}

We thank Walter Richtering for sharing laboratory space and equipment. Walter Richtering, Dmitry Pergushov and Andreas Schmid are acknowledged for fruitful discussions. We are very much thankful to Toni Gossen for his help with NMR. Further, we appreciate the proofreading of Olga Mergel and Natascha Drude. Finally, we are indebted to the Fund of the German Chemical Industry (FCI) and to the German Research Foundation (DFG) for financial assistance (PL 571/ 3-1 and RI 560/20-1). 


\section{Notes and references}

1 A. Heise, J. L. Hedrick, C. W. Frank and R. D. Miller, J. Am. Chem. Soc., 1999, 121, 8647-8648.

2 J. Park, M. Moon, M. Seo, H. Choi and S. Y. Kim, Macromolecules, 2010, 43, 8304-8313.

3 N. Stavrouli, A. I. Triftaridou, C. S. Patrickios and C. Tsitsilianis, Macromol. Rapid Commun., 2007, 28, 560-566.

4 S. Luo, J. Xu, Z. Zhu, C. Wu and S. Liu, J. Phys. Chem. B, 2006, 110, 9132-9139.

5 J. Xu, S. Luo, W. Shi and S. Liu, Langmuir, 2006, 22, 989-997.

6 M. Rasa, M. A. R. Meier and U. S. Schubert, Macromol. Rapid Commun., 2007, 28, 1429-1433.

7 T. Satoh, Soft Matter, 2009, 5, 1972-1982.

8 D. Kul, L. M. Van Renterghem, M. A. R. Meier, S. Strandman, H. Tenhu, S. S. Yilmaz, U. S. Schubert and F. E. Du Prez, J. Polym. Sci., Part A: Polym. Chem., 2007, 46, 650-660.

9 D. Kuckling and A. Wycisk, J. Polym. Sci., Part A: Polym. Chem., 2013, 51, 2980-2994.

10 R. K. Kainthan, C. Mugabe, H. M. Burt and D. E. Brooks, Biomacromolecules, 2008, 9, 886-895.

11 G. Cheng, A. Böker, M. Zhang, G. Krausch and A. H. E. Müller, Macromolecules, 2001, 34, 6883-6888.

12 J. Selb and Y. Gallot, Makromol. Chem., 1981, 182, 1775-1786.

13 A. Kikuchi and T. Nose, Macromolecules, 1996, 29, 6770-6777.

14 S.-i. Yusa, A. Sakakibara, T. Yamamoto and Y. Morishima, Macromolecules, 2002, 35, 5243-5249.

15 Y. Zhou, K. Jiang, Q. Song and S. Liu, Langmuir, 2007, 23, 13076-13084.

16 S. Yusa, A. Sakakibara, T. Yamamoto and Y. Morishima, Macromolecules, 2002, 35, 10182-10188.

17 S. Strandman, S. Hietala, V. Aseyev, B. Koli, S. J. Butcher and H. Tenhu, Polymer, 2006, 47, 6524-6535.

18 S. Strandman, A. Zarembo, A. A. Darinskii, P. Laurinmaki, S. J. Butcher, E. Vuorimaa, H. Lemmetyinen and H. Tenhu, Macromolecules, 2008, 41, 8855-8864.

19 X. S. Wang, M. A. Winnik and I. Manners, Macromol. Rapid Commun., 2003, 24, 403-407.

20 A. A. Steinschulte, B. Schulte, M. Erberich, O. V. Borisov and F. A. Plamper, ACS Macro Lett., 2012, 1, 504-507.

21 A. P. Gelissen, D. V. Pergushov and F. A. Plamper, Polymer, 2013, 54, 6877-6881.

22 Y. Chang, W.-C. Chen, Y.-J. Sheng, S. Jiang and H.-K. Tsao, Macromolecules, 2005, 38, 6201-6209.

23 C.-M. Lin, Y.-Z. Chen, Y.-J. Sheng and H.-K. Tsao, React. Funct. Polym., 2009, 69, 539-545.

24 V. Aseyev, H. Tenhu and F. M. Winnik, Adv. Polym. Sci., 2011, 242, 29-89.

25 S. Hocine and M.-H. Li, Soft Matter, 2013, 9, 5839-5861.

26 G. Marinov, B. Michels and R. Zana, Langmuir, 1998, 14, 2639-2644.

27 J. Kriz and J. Dybal, J. Phys. Chem. B, 2010, 114, 3140-3151.

28 S. O. Kyeremateng, K. Busse, J. Kohlbrecher and J. Kressler, Macromolecules, 2011, 44, 583-593.

29 L. Bromberg, S. Deshmukh, M. Temchenko, L. Iourtchenko, V. Alakhov, C. Alvarez-Lorenzo, R. Barreiro-Iglesias,
A. Concheiro and T. A. Hatton, Bioconjugate Chem., 2005, 16, 626-633.

30 F. A. Plamper, M. Ruppel, A. Schmalz, O. Borisov, M. Ballauff and A. H. E. Müller, Macromolecules, 2007, 40, 8361-8366.

31 F. A. Plamper, J. R. McKee, A. Laukkanen, A. Nykänen, A. Walther, J. Ruokolainen, V. Aseyev and H. Tenhu, Soft Matter, 2009, 5, 1812-1821.

32 F. A. Plamper, L. Murtomäki, A. Walther, K. Kontturi and H. Tenhu, Macromolecules, 2009, 42, 7254-7257.

33 F. A. Plamper, S. Reinicke, M. Elomaa, H. Schmalz and H. Tenhu, Macromolecules, 2010, 43, 2190-2203.

34 X. Sun, G. Jiang, Y. Wang and Y. Xu, Colloid Polym. Sci., 2011, 289, 677-684.

35 A. M. Alhoranta, J. K. Lehtinen, A. O. Urtti, S. J. Butcher, V. O. Aseyev and H. J. Tenhu, Biomacromolecules, 2011, 12, 3213-3222.

36 J. Niskanen, M. Karesoja, T. Rossi and H. Tenhu, Polym. Chem., 2011, 2, 2027-2036.

37 T. Takagishi, T. Hosokawa and Y. Hatanaka, J. Polym. Sci., Part A: Polym. Chem., 1989, 27, 1-13.

38 A. Housni and Y. Zhao, Langmuir, 2010, 26, 12933-12939.

39 W. S. Tan, R. E. Cohen, M. F. Rubner and S. A. Sukhishvili, Macromolecules, 2010, 43, 1950-1957.

40 P. Petrov, C. B. Tsvetanov and R. Jerome, J. Phys. Chem. B, 2009, 113, 7527-7533.

41 A. Vesterinen, S. Lipponen, J. Rich and J. Seppälä, eXPRESS Polym. Lett., 2011, 5, 754-765.

42 A. Steinschulte, B. Schulte, N. Drude, M. Erberich, C. Herbert, J. Okuda, M. Möller and F. Plamper, Polym. Chem., 2013, 4, 3885-3895.

43 S. Inal, L. Chiappisi, J. D. Kölsch, M. Kraft, M.-S. Appavou, U. Scherf, M. Wagner, M. R. Hansen, M. Gradzielski, A. Laschewsky and D. Neher, J. Phys. Chem. B, 2013, 117, 14576-14587.

44 K. Kalyanasundaram and J. K. Thomas, J. Am. Chem. Soc., 1977, 99, 2039-2044.

45 Y. Diaz-Fernandez, F. Foti, C. Mangano, P. Pallavicini, S. Patroni, A. Perez-Gramatges and S. Rodriguez-Calvo, Chem.-Eur. J., 2006, 12, 921-930.

46 J. Virtanen, H. Lemmetyinen and H. Tenhu, Polymer, 2001, 42, 9487-9493.

47 J. Virtanen, S. Holappa, H. Lemmetyinen and H. Tenhu, Macromolecules, 2002, 35, 4763-4769.

48 F. A. Plamper, A. A. Steinschulte, C. H. Hofmann, N. Drude, O. Mergel, C. Herbert, M. Erberich, B. Schulte, R. Winter and W. Richtering, Macromolecules, 2012, 45, 8021-8026.

49 R. Michels, T. Sinemus, J. Hoffmann, B. Brutschy and K. Huber, J. Phys. Chem. B, 2013, 117, 8611-8619.

50 A. Sehgal and T. A. P. Seery, Macromolecules, 1998, 31, 7340-7346.

51 A. Mei, X. Guo, Y. Ding, X. Zhang, J. Xu, Z. Fan and B. Du, Macromolecules, 2010, 43, 7312-7320.

52 X. Liang, C. Guo, J. Ma, J. Wang, S. Chen and H. Liu, J. Phys. Chem. B, 2007, 111, 13217-13220.

53 J. Yan, W. Ji, E. Chen, Z. Li and D. Liang, Macromolecules, 2008, 41, 4908-4913. 
54 O. Casse, A. Shkilnyy, J. Linders, C. Mayer, D. Haussinger, A. Völkel, A. F. Thünemann, R. Dimova, H. Cölfen, W. Meier, H. Schlaad and A. Taubert, Macromolecules, 2012, 45, 4772-4777.

55 W. F. Polikt and W. Burchard, Macromolecules, 1983, 16, 978-982.

56 M. J. N. Junk, W. Li, A. D. Schlüter, G. Wegner, H. W. Spiess, A. Zhang and D. Hinderberger, Macromol. Chem. Phys., 2011, 212, 1229-1235.

57 M. Daoud and J. P. Cotton, J. Phys., 1982, 43, 531-538.

58 J. S. Pedersen and M. C. Gerstenberg, Macromolecules, 1996, 29, 1363-1365.

59 S. Manet, A. Lecchi, M. Imperor-Clerc, V. Zholobenko, D. Durand, C. L. P. Oliveira, J. S. Pedersen, I. Grillo, F. Meneau and C. Rochas, J. Phys. Chem. B, 2011, 115, 11318-11329.

60 P. Wu and L. Brand, Anal. Biochem., 1994, 218, 1-13.
61 F. A. Plamper, A. P. Gelissen, J. Timper, A. Wolf, A. B. Zezin, W. Richtering, H. Tenhu, U. Simon, J. Mayer, O. V. Borisov and D. V. Pergushov, Macromol. Rapid Commun., 2013, 34, $855-860$.

62 Y. Xu, F. Plamper, M. Ballauff and A. H. E. Müller, Adv. Polym. Sci., 2010, 228, 1-38.

63 M. Tricot, Macromolecules, 1984, 17, 1698-1704.

64 S. V. Larin, A. A. Darinskii, E. B. Zhulina and O. V. Borisov, Langmuir, 2009, 25, 1915-1918.

65 E. B. Zhulina and O. V. Borisov, Macromolecules, 2008, 41, 5934-5944.

66 F. A. Plamper, H. Becker, M. Lanzendörfer, M. Patel, A. Wittemann, M. Ballauff and A. H. E. Müller, Macromol. Chem. Phys., 2005, 206, 1813-1825.

67 H. Kojima and F. Tanaka, J. Polym. Sci., Part B: Polym. Phys., 2013, 51, 1112-1123. 